\title{
Curcumin-loaded magnetic nanoparticles for breast cancer therapeutics and imaging applications
}

This article was published in the following Dove Press journal:

International Journal of Nanomedicine

31 March 2012

Number of times this article has been viewed

\author{
Murali M Yallapu' \\ Shadi F Othman ${ }^{2}$ \\ Evan T Curtis ${ }^{2}$ \\ Nichole A Bauer' \\ Neeraj Chauhan ${ }^{1,3}$ \\ Deepak Kumar ${ }^{4,5}$ \\ Meena Jaggi ${ }^{1,3,6}$ \\ Subhash C Chauhan 1,3,6 \\ 'Cancer Biology Research Center, \\ Sanford Research/University of South \\ Dakota, Sioux Falls, SD, ${ }^{2}$ Department \\ of Biological Systems Engineering, \\ University of Nebraska-Lincoln, \\ Lincoln, NE, ${ }^{3}$ Basic Biomedical \\ Science Division, Sanford School of \\ Medicine, University of South Dakota, \\ Sioux Falls, SD, ${ }^{4}$ Cancer Research \\ Laboratory, Department of Biology, \\ University of the District of Columbia, \\ ${ }^{5}$ Lombardi Comprehensive Cancer \\ Center, Georgetown University, \\ Washington, DC, ${ }^{6}$ Department of \\ Obstetrics/Gynecology, Sanford \\ School of Medicine, University of \\ South Dakota, Sioux Falls, SD, USA
}

Correspondence: Subhash C Chauhan Cancer Biology Research Center, Sanford Research/University of South Dakota, 230I E. 60th Street North, Sioux Falls, SD 57I04-0589, USA

$\mathrm{Tel}+\mathrm{I} 6053126106$

Fax + I 605 3/2 607|

Email subhash.chauhan@sanfordhealth.org
Background: The next generation magnetic nanoparticles (MNPs) with theranostic applications have attracted significant attention and will greatly improve nanomedicine in cancer therapeutics. Such novel MNP formulations must have ultra-low particle size, high inherent magnetic properties, effective imaging, drug targeting, and drug delivery properties. To achieve these characteristic properties, a curcumin-loaded MNP (MNP-CUR) formulation was developed. Methods: MNPs were prepared by chemical precipitation method and loaded with curcumin (CUR) using diffusion method. The physicochemical properties of MNP-CUR were characterized using dynamic light scattering, transmission electron microscopy, and spectroscopy. The internalization of MNP-CUR was achieved after 6 hours incubation with MDA-MB-231 breast cancer cells. The anticancer potential was evaluated by a tetrazolium-based dye and colony formation assays. Further, to prove MNP-CUR results in superior therapeutic effects over CUR, the mitochondrial membrane potential integrity and reactive oxygen species generation were determined. Magnetic resonance imaging capability and magnetic targeting property were also evaluated.

Results: MNP-CUR exhibited individual particle grain size of $\sim 9 \mathrm{~nm}$ and hydrodynamic average aggregative particle size of $\sim 123 \mathrm{~nm}$. Internalized MNP-CUR showed a preferential uptake in MDA-MB-231 cells in a concentration-dependent manner and demonstrated accumulation throughout the cell, which indicates that particles are not attached on the cell surface but internalized through endocytosis. MNP-CUR displayed strong anticancer properties compared to free CUR. MNP-CUR also amplified loss of potential integrity and generation of reactive oxygen species upon treatment compared to free CUR. Furthermore, MNP-CUR exhibited superior magnetic resonance imaging characteristics and significantly increased the targeting capability of CUR

Conclusion: MNP-CUR exhibits potent anticancer activity along with imaging and magnetic targeting capabilities. This approach can be extended to preclinical and clinical use and may have importance in cancer treatment and cancer imaging in the future. Further, if these nanoparticles can functionalize with antibody/ligands, they will serve as novel platforms for multiple biomedical applications.

Keywords: magnetic nanoparticles, drug delivery systems, magnetic resonance imaging, nanomedicine, cancer therapeutics, biomedical applications

\section{Introduction}

Breast cancer is a commonly diagnosed cancer among women in the United States. It is estimated that 230,480 women were diagnosed with, and 39,520 women died from, breast cancer in 2011 in the United States. ${ }^{1}$ Basal-like breast cancer accounts for $15 \%$ of all breast cancers and has a poor prognosis. The majority of these cancers are referred to as triple-negative breast cancers because they do not over express estrogen 
receptor, progesterone receptor, or human epidermal growth factor receptor 2.2,3 Chemotherapy is an effective option to treat such cancers; however, chemotherapy is always associated with several sets of side effects. ${ }^{4-7}$ To overcome/avoid issues with side effects, natural compounds which are used as daily food ingredients may be an alternative option.

Among many food ingredients, curcumin (CUR), a natural diphenol extracted from the ground rhizomes of Curcuma longa, possesses a number of potentially beneficial biological characteristics including anticancer and chemoprevention properties. ${ }^{8}$ MDA-MB-231 cancer cells are one example of a triple-negative breast cancer and are highly metastatic in nature. CUR can effectively upregulate p53, p21, and p27 and downregulate cyclin E; thus, cell cycle arrest occurs at the G1 phase in these cancer cells. ${ }^{9}$ CUR also decreases cell viability and anchorage independency of MDA-MB-231 cancer cells by induction of apoptosis and inhibits constitutive STAT3 signaling..$^{10}$ Another study confirmed that CUR enhances cellular localization and phosphorylation, induces DNA damage and modulates BRCA1 in triple-negative breast cancer cells. ${ }^{11}$ Additionally, synergistic antiproliferation effects of CUR were observed when used in conjunction with chemotherapeutic drugs or radiation. ${ }^{12-14}$ A recent review article suggests the specific role of CUR towards cancer cells versus normal cells. ${ }^{15}$ Specifically, CUR does not show significant toxicity towards normal human cells. However, successful implementation of CUR into the clinical arena must overcome issues related to its solubility, degradation in physiological medium, and rapid metabolism. ${ }^{16}$ This can be achieved through advanced drug delivery systems. ${ }^{16,17}$ Recent studies demonstrate CUR entrapped or encapsulated in nanocarriers, such as polymer nanoparticles, nanoassemblies, and self-assemblies, can enhance internalization/localization and thus induce cytotoxicity and apoptosis in cancer cells. ${ }^{18-22}$ Similarly, CUR nanoformulations (nanomedicine of CUR) based on conjugate, emulsion, lipid, polymer, and gel nanoparticles have been proposed for improving therapeutic benefits and sensitization for chemotherapy and radiation. ${ }^{16,17,23}$

Magnetic nanoparticles (MNPs; mostly iron oxide nanoparticles) are widely used in various medical applications. MNPs have been utilized as nanocarriers for drugs, contrast imaging agents in magnetic resonance imaging (MRI), in local hyperthermia, and magnetic targeting. ${ }^{24,25}$ However, high aggregation of MNPs is a common problem. High surface-to-volume ratios and van der Waals forces trigger opsonization and therefore, present a major obstacle for biomedical applications. This phenomenon can be altered by engineering the surface of the MNPs through various stabilizer coatings including surfactants and synthetic and natural polymers. ${ }^{25}$ Additionally, some reports suggest that some polymer coatings on the surface of MNPs can change the contrast imaging properties appropriate for both $\mathrm{T}_{1}$ and $\mathrm{T}_{2}$ imaging. ${ }^{26}$

Advances in cancer nanotechnology increase the use of novel therapeutic strategies such as nanotheranostics, which utilize individualized diagnostic therapy. ${ }^{27}$ The interest here was to develop a magnetic nanoformulation without compromising its basic imaging and drug delivery characteristics. To date, a wide variety of modalities have been formulated with MNP platforms that contribute not only to drug delivery but also exhibit photothermal therapy, thermosensitive chemotherapy, and visible/luminescence/near infrared/multimodal imaging. ${ }^{26,28,29}$ Such nanoformulation applicability relies on the design and stabilizer coatings which provide biocompatibility, high therapeutic drug loading capability, stability in biological milieu, and proper intracellular uptake by cancer cells. Crosslinked dextrancoated MNPs are recognized as an example of a nanoplatform with multiple functions. ${ }^{30}$ The engineered structure of this formulation maintains a targeting feature and diagnostic imaging properties for MRI, optical, and positron emission tomography modalities. Many important formulations have been developed and the iron oxide nanocore stabilized with a double layer coating has frequently proved to be feasible for theranostic purposes. ${ }^{31,32}$ However, these formulations usually exhibit a higher hydrodynamic diameter in aqueous medium ( $\geq 250 \mathrm{~nm}$ ) which limits their use as efficient therapeutic carriers for medicinal applications. Therefore, herein, a rationally designed MNP with improved features was developed. This formulation was composed of an iron oxide core coated with $\beta$-cyclodextrin (CD) and pluronic F68 polymer (polyethylene oxide-co-polypropylene oxide-copolyethylene oxide) that allows loading of anticancer drugs (in this study, CUR). This formulation was examined for its anticancer properties, magnetic targeting, and MRI in triplenegative breast cancer (MDA-MB-231) cells.

\section{Materials and methods Chemicals and cell culture}

All chemicals and reagents were purchased from SigmaAldrich Corporation (St Louis, MO) unless otherwise specified. Difco ${ }^{\circledR}$ bacto-nutrient agar dehydrated was purchased from BD Diagnostics (Franklin Lakes, NJ). Milli-Q ${ }^{\circledR}$ purified water (Millipore Corporation, Billerica, MA) was used to make all aqueous solutions. MDA-MB-231 
breast cancer cells were kindly gifted by Dr W Keith Miskimins (Cancer Biology Research Center, Sanford Research/University of South Dakota, Sioux Falls, SD). This cell line was maintained as monolayer culture in Dulbecco's modified Eagle's medium (high glucose; Thermo Scientific HyClone, Logan, UT) supplemented with 10\% fetal bovine serum (Atlanta Biologicals Inc, Lawrenceville, GA) and Gibco $^{\circledR} 1 \%$ penicillin-streptomycin (Invitrogen Life Technologies, Carlsbad, CA) at $37^{\circ} \mathrm{C}$ in a humidified atmosphere ( $5 \%$ carbon dioxide).

\section{Preparation of MNP and MNP-CUR formulations}

Iron oxide nanoparticles were prepared using a minor modification of a protocol reported by Yallapu et al. ${ }^{22}$ Briefly, $100 \mathrm{mg}$ of CD and $45 \mathrm{~mL}$ of water containing $810 \mathrm{mg}$ of iron(3+) and $297 \mathrm{mg}$ of iron(2+) ions (molar ratio 2:1) was placed in a $100 \mathrm{~mL}$ beaker. To this solution, $3 \mathrm{~mL}$ of ammonium hydroxide (28\% ammonia in water) was slowly added at a stirring speed of $900 \mathrm{rpm}$. After 6 hours, $200 \mathrm{mg}$ of pluronic polymer F68 (average molecular weight 8350) in $5 \mathrm{~mL}$ water was added to the nanoparticle suspension and stirred overnight. After five washes with water, ${ }^{33}$ the nanoparticles were resuspended in $25 \mathrm{~mL}$ water and centrifuged at $1000 \mathrm{rpm}$ to remove larger aggregates. This formulation was designated as MNP.

To load CUR in the MNPs, $1 \mathrm{mg}$ CUR in $200 \mu \mathrm{L}$ acetone was added dropwise to $2.75 \mathrm{~mL}$ of an aqueous dispersion of MNPs (10 mg). The mixture was stirred overnight at 400 rpm on a magnetic plate to facilitate the penetration of CUR molecules into the $\mathrm{CD}$ or $\mathrm{CD}-\mathrm{F} 68$ polymer layers in the formulation. The drug-loaded nanoparticles were washed three times by resuspending them in water and separated with the help of magnets. ${ }^{33}$ These drug-loaded nanoparticles were dispersed in $2 \mathrm{~mL}$ sterile phosphate buffered saline (PBS) solution and stored in a refrigerator until further use. This formulation was designated as MNP-CUR. The CUR loading estimation was determined using an ultraviolet-visible (UV-Vis) spectrophotometer (BioMate ${ }^{\mathrm{TM}} 3$; Thermo Fisher Scientific, Pittsburgh, PA) at $450 \mathrm{~nm}$, following a previously reported procedure. ${ }^{18,22}$

\section{Characterization}

A transmission electron microscope (TEM) (1210; JEOL Ltd, Tokyo, Japan) was employed to determine the size and morphology of the MNP-CUR nanoparticles. For this study, a sample was prepared by placing the nanoparticle suspension ( $50 \mu \mathrm{L}$ of $1 \mathrm{mg} / \mathrm{mL}$ ) on a 200 mesh formvar-coated copper
TEM grid (grid size: $97 \mu \mathrm{m}$; Ted Pella Inc, Redding, CA). The excess was removed using a piece of fine filter paper and the grid was allowed to air dry prior to imaging under the microscope operating at $80 \mathrm{kV}$.

The particle size, distribution, and zeta potential in aqueous medium of the MNP-CUR formulation $(50 \mu \mathrm{L}$ of $500 \mu \mathrm{g} / \mathrm{mL}$ dispersed in $3 \mathrm{~mL}$ of distilled water and ultrasonicated for 30 minutes) were determined by employing a Delsa ${ }^{\mathrm{TM}}$ Nano $\mathrm{C}$ particle size analyzer (Beckman Coulter, Brea, CA). Particle size and distribution was measured for 3 minutes, and the zeta potential was measured for 90 runs (9 minutes).

Fourier transform infrared (FTIR) spectral study was conducted for dry MNP and MNP-CUR (obtained by lyophilization using a Labconco Freeze Dry System, $-48^{\circ} \mathrm{C}$, $133 \times 10^{-3}$ mbar; Labconco Corporation, Kansas City, MO). The FTIR of nanoparticles was recorded at a scanning speed of $4 \mathrm{~cm}^{-1}$ between $4000-750 \mathrm{~cm}^{-1}$ (32 scans) on an IlluminatIR ${ }^{\mathrm{TM}}$ FT-IR microscope (Smiths Detection, Danbury, CT).

\section{In vitro CUR release}

CUR release from the MNP-CUR formulation was carried out in a PBS (154 mM, pH 7.4) solution containing $0.1 \%$ weight/volume Tween- $80^{\circledR}$ (Sigma-Aldrich) at $37^{\circ} \mathrm{C}$ at 100 rpm in an orbital shaker (Max $\mathrm{Q}^{\mathrm{TM}} 4000$ series; Thermo Fisher). Briefly, $2 \mathrm{mg}$ of MNP-CUR was suspended in $3 \mathrm{~mL}$ PBS solution in a $5 \mathrm{~mL}$ tube. At predetermined intervals, the tube was centrifuged at 10,000 rpm for 30 minutes and supernatant was stored at $4^{\circ} \mathrm{C}$ in the dark. Then fresh buffer solution was replaced in the tube to redisperse the pellet. The collected supernatants were measured for absorbance at $450 \mathrm{~nm}$ using a UV-Vis spectrometer as described earlier. ${ }^{22}$ An identical standard of CUR $(0-10 \mu \mathrm{g} / \mathrm{mL})$ was prepared and absorbance was measured at $450 \mathrm{~nm}$ using the UV-Vis spectrometer to estimate the amount of CUR released from MNP-CUR.

\section{Nanoparticles cellular uptake}

A stable nanoformulation is determined by its cellular uptake which is one of the important parameters for drug delivery applications. To verify this phenomenon, the uptake capacity of MNP-CUR formulation was evaluated in MDA-MB-231 cancer cells. Cells $\left(5 \times 10^{5}\right)$ were seeded in six-well plates in $2 \mathrm{~mL}$ medium. After 24 hours, media was replaced with medium containing 0-30 $\mu \mathrm{M}$ MNP-CUR. After 6 hours incubation time, cells were washed twice with PBS and stained with Prussian blue to detect iron presence 
in the cancer cells. ${ }^{34}$ Cell images were captured using an EVOS $^{\circledR}$ phase contrast microscope (Advanced Microscopy Group, Bothell, WA).

To further validate this cellular uptake (internalization) behavior of MNP-CUR, TEM was employed. This study provides clear evidence of the presence of nanoparticle internalization at higher magnification. For this experiment, $1 \times 10^{7}$ cells per $150 \mathrm{~mm}$ plate were incubated with $20 \mu \mathrm{M}$ of MNP-CUR in $20 \mathrm{~mL}$ media for 6 hours. These cells were centrifuged to remove unbound or uninternalized nanoparticles, fixed with standard ice cold formaldehyde (4\%)-glutaraldehyde (1\%) fixative solution followed by osmium tetroxide fixative solution, and imaged under TEM, following a previously published method. ${ }^{22}$

\section{Protein adsorption}

The protein adsorption on the surface of MNP-CUR was evaluated following a developed protocol. ${ }^{35}$ For this ex vivo study, $20 \mu \mathrm{L}$ of human or mouse serum was added to MNP-CUR formulation ( $1 \mathrm{mg} / \mathrm{mL}$ ) and stirred for 2 hours at $25^{\circ} \mathrm{C}$. The serum-bound MNP-CUR formulation pellet was collected after centrifugation at 10,000 rpm for 30 minutes. The MNP-CUR pellet was redispersed in $3 \mathrm{~mL}$ water and measured for particle size using a particle size analyzer and TEM as described above.

\section{In vitro cytotoxicity}

Methyl tetrazolium salt (MTS) assay

To assess in vitro cytotoxicity of CUR and MNP-CUR, a standard MTS (3-[4,5-dimethyl-2-yl]-5-[3-carboxymethoxyphenyl]-2-[4-sulfophenyl]-2H-tetrazolium) cell proliferation assay (CellTiter 96 ${ }^{\circledR}$ AQueous; Promega Corporation, Madison, WI) was employed. For this assay, MDA-MB-231 cells (5000 cells/well in $100 \mu \mathrm{L}$ media) were seeded in 96-well plates and allowed to attach overnight. Cells were then incubated with desired concentrations $(5-40 \mu \mathrm{M})$ of CUR/MNP-CUR for 2 days. Equivalent amounts of dimethyl sulfoxide (DMSO) or MNP in PBS served as controls for this experiment. After 2 days, the media was replaced with fresh media $(100 \mu \mathrm{L})$ and MTS reagent $(25 \mu \mathrm{L})$ in each well. After 2 hours, the color intensity resulting from the formation of soluble formazan product was measured at $492 \mathrm{~nm}$ using a microplate reader (BioMate $3 \mathrm{UV}-\mathrm{Vis}$ spectrophotometer). This absorption reading is directly proportional to the number of live cells in the culture. The percentage of cell growth was presented as the percentage of the absorption of treated cells to the absorption of nontreated cells. Data presented are from six replicates.

\section{Colony formation assay}

Colony formation assay was employed to determine the longterm anticancer potential of the CUR/MNP-CUR formulation. In brief, MDA-MB-231 cancer cells were seeded at a density of 1000 per well in six-well plates in $2 \mathrm{~mL}$ medium and allowed 2-3 days to begin formation of colonies. Then, cells were treated with different concentrations $(4-8 \mu \mathrm{M})$ of CUR or MNP-CUR over a period of 10 days. The plates were washed three times with PBS, fixed in chilled methanol, stained with hematoxylin (Thermo Fisher), washed with tap water and air dried. The density of colonies was counted according to a previous method using a Multimage ${ }^{\text {TM }}$ light cabinet (Alpha Innotech Corporation, San Leandro, CA) with the help of AlphaEaseFC ${ }^{\text {TM }}$ (Alpha ImagerHP AIC) software. ${ }^{18,21}$ The percent density of colonies was calculated using the density of colonies formed in treatment divided by the density of colonies formed in DMSO or MNP in PBS. Data presented are from triplicate studies.

\section{Mitochondrial membrane potential}

The loss of mitochondrial membrane potential is a direct indication of the apoptosis. This can be detected by a cationic fluorescent red-orange dye, tetramethyl rhodamine ethyl ester (TMRE; Invitrogen). For this experiment, MDAMB-231 cells $\left(1 \times 10^{6}\right)$ were seeded in a $100-\mathrm{mm}$ Petri dish and allowed to attach overnight. These cells were exposed to medium containing $5 \mu \mathrm{M}$ and $10 \mu \mathrm{M}$ CUR or MNP-CUR and incubated for 24 hours at $37^{\circ} \mathrm{C}$. Cells treated with DMSO and MNPs without CUR in medium served as controls. The cells were then washed twice with PBS and incubated with $100 \mathrm{nM}$ TMRE for 20 minutes at $37^{\circ} \mathrm{C}$ in medium without phenol red. After completion of the incubation period, cells were washed twice with PBS, trypsinized, and collected in $1 \mathrm{~mL}$ medium containing hydroxyethyl piperazineethanesulfonic acid without phenol red. Cell images were captured using an Olympus $^{\circledR} 1$ X71 microscope equipped with a DP71 camera (Olympus Corporation, Tokyo, Japan). Quantitative TMRE fluorescence stain in mitochondria was detected by injecting $25 \mu \mathrm{L}$ cell suspension into an Accuri ${ }^{\circledR}$ C6 flow cytometer (BD Accuri Cytometers, Ann Arbor, MI) to determine the mean fluorescence levels in the FL2 channel. The experiment was conducted in triplicate.

\section{Reactive oxygen species (ROS)}

For this experiment, MDA-MB-231 cells $\left(1 \times 10^{6}\right)$ were plated in a $100-\mathrm{mm}$ Petri dish and allowed to attach overnight. Then the medium was replaced with medium containing $5 \mu \mathrm{M}$ and $10 \mu \mathrm{M}$ CUR or MNP-CUR and 
incubated for 24 hours at $37^{\circ} \mathrm{C}$. Cells treated with DMSO and nanoparticles without CUR in medium served as controls. After that, these cells were washed twice with PBS and treated with $5 \mu \mathrm{M}$ MitoSOX ${ }^{\mathrm{TM}}$ Red mitochondrial superoxide indicator (Invitrogen) for 20 minutes at $37^{\circ} \mathrm{C}$ in medium without phenol red. MitoSOX is rapidly oxidized by superoxide and this complex is highly fluorescent upon binding to nucleic acid. Cell images were taken using an Olympus 1X71 microscope equipped with a DP71 camera. The production of ROS was detected by injecting $25 \mu \mathrm{L}$ cell suspension into an Accuri C6 flow cytometer to determine the mean fluorescence levels in the FL2 channel. The experiment was conducted in triplicate.

\section{In vitro $\mathrm{MRI}$}

MNP and MNP-CUR formulations were diluted in 3\% agar solution and final concentration of formulations in agar solution was kept between 10-40 $\mu \mathrm{g}$ iron/mL. MRI experiments were performed at $25^{\circ} \mathrm{C}$ with a $9.4 \mathrm{~T}\left(400 \mathrm{MHz} \mathrm{H}^{1}\right)$, $89 \mathrm{~mm}$ vertical bore MRI scanner equipped with triple axis gradients $(100 \mathrm{G} / \mathrm{cm})$ and a $4-\mathrm{cm}$ Millipede transmit/receive radiofrequency coil (Agilent Technologies, Santa Clara, CA). Tubes containing formulations in agar solution were placed into the MRI scanner and a number of spin-echo sequences were run to determine both $T_{1}$ and $T_{2}$ relaxation times. $T_{2}$ relaxation time was measured using a spin-echo nuclear magnetic resonance spectra analysis sequence with 32 echo times arrayed exponentially from 5-300 milliseconds and the repetition time of 8000 milliseconds. Similarly, the $T_{1}$ relaxation time was measured by varying repetition times between 100-6400 milliseconds while keeping the echo time constant at 8 milliseconds. Relaxation rates $\mathrm{R}_{1}\left(1 / \mathrm{T}_{1}\right)$ and $\mathrm{R}_{2}\left(1 / \mathrm{T}_{2}\right)$ were calculated by monoexponential curve fitting of the signal intensity versus time (echo time or repetition time) using Origin ${ }^{\circledR} 6.1$ software (OriginLab Corporation, Northampton, MA). Equations (1) and (2) were used for curve fitting of relaxation rates $R_{1}$ and $R_{2}$, respectively: ${ }^{36}$

$$
\begin{aligned}
& R_{1}=A \times\left[1-\exp \left(-R_{1} \times T R\right)\right] \\
& R_{2}=A+C \times\left[\exp \left(-R_{2} \times T E\right)\right]
\end{aligned}
$$

$\mathrm{T}_{1}$ relaxivity $\left(\mathrm{r}_{1}\right)$ and $\mathrm{T}_{2}$ relaxivity $\left(\mathrm{r}_{2}\right)$ were calculated from the slope of plot of $R_{1}$ versus iron concentration and $R_{2}$ versus iron concentration in agar gels, respectively. TR represents repetition time and TE represents echo time.

\section{Magnetic targeting}

Magnet-mediated delivery, ie, magnetic drug targeting, can increase cellular uptake of nanoparticles in cancer cells and improve localized delivery of the encapsulated drug. For magnetic drug targeting, MDA-MB-231 cells $\left(2 \times 10^{5}\right)$ were seeded in six-well plates and allowed to attach overnight. The following day, cells were treated for 3 hours with 2.5, 5, and $7.5 \mu \mathrm{M}$ of CUR or MNP-CUR or MNP-CUR in the presence of a neodymium external magnet ( 1 " Dia x 3/32" thick Rare Earth Magnet Disc; Amazing Magnets, Irvine, CA). Cells treated with DMSO, MNP, and MNP with magnet served as controls. Cells were then washed with PBS three times to remove unbound and uninternalized CUR/MNP-CUR. Medium without phenol red was added to the dishes to image using a fluorescence microscope (Olympus $1 \times 71$ ). After imaging, cells were collected in $1 \mathrm{~mL}$ medium by harvesting with trypsin and analyzed for semiqualitative cellular uptake of CUR and MNP-CUR using flow cytometry. ${ }^{19,21,22}$ The inherent fluorescence property of CUR was utilized to evaluate cellular uptake.

\section{Statistical analysis}

The data was processed using Microsoft Excel 2007 software and presented as mean \pm standard error of the mean. Statistical analyses were performed using an unpaired, two-tailed Student's $t$-test. The level of significance was set at $* P<0.05$. All graphs were plotted using Origin 6.1 software.

\section{Results and discussion}

Nanotheranostics is a multidisciplinary approach in medicine composed of therapy and imaging/diagnostic applications. ${ }^{25,26}$ To achieve such multiple functions in a single nanoformulation, a multidisciplinary collaborative expertise involving chemistry, drug delivery, materials science, and biological sciences is required. MNPs are considered one of the important materials for theranostic applications due to their inherent low particle size, high magnetization values, biocompatibility, and ability to load biomolecules or drug molecules. ${ }^{24}$ Therefore, development of monodispersed MNPs is essential for biomedical applications. Numerous methods have been developed following various coating approaches to fabricate magnetic nanoformulations with well-defined particle size and shape. ${ }^{37,38}$ These approaches still need to overcome destabilization mechanisms such as Ostwald ripening, flocculation, phase inversion, and creaming. To improve the features of these magnetic nanoformulations, a simple precipitation approach was designed where an iron oxide nanoparticle core was prepared in the presence of $\mathrm{CD}$ and subsequently coated with pluronic polymer F68 (poly[ethylene-co-propylene glycol]) (Figure A1, Appendix). CD selection in this formulation was based 
upon its combination of hydrophilic units $(-\mathrm{OH})$ which can bind to the iron oxide nanoparticle surface and is capable of including anticancer $\operatorname{drug}(\mathrm{s})$ via the hydrophobic cavity. ${ }^{21,39}$ Additional coating with F68 polymer results in a hydrophobic (polypropylene, polypropylene oxide) chain which can bind to the hydrophobic cavities of CD and the hydrophilic (polyethylene glycol, polyethylene oxide) chain provides additional hydrophilicity and stability to particles. ${ }^{22,40,41}$ $\mathrm{CD}$ and F68 polymer coating resulted in reduction of the nanoparticle aggregation phenomenon (Figure A1, Appendix). Similar patterns of decreased nanoparticle aggregation were observed in dynamic light scattering particle size analysis, ie, the aggregative particle size of nanoparticles decreased from $208 \mathrm{~nm}$ to $116 \mathrm{~nm}$ (Figure A1, Appendix). However, the size of the individual nanoparticle grain remained unchanged. The diameter of particles observed in TEM was smaller than that detected by the dynamic light scattering method because TEM observations were of the nanoparticles in dry form while dynamic light scattering observations were of the nanoparticles in aqueous suspensions.
Figure 1A illustrates the hypothetical single nanoparticle layer structure of MNP-CUR formulation where CUR drug molecules are positioned in CD layer(s). The TEM image of MNP-CUR demonstrates individual nanoparticle grains had an average size of about $9 \mathrm{~nm}$ (Figure 1B). Dynamic light scattering data of MNP-CUR reveals the particles aggregative size averaged $123 \mathrm{~nm}$ (Figure 1C) with unimodal and narrow size distribution, and contained a polydispersity index of 0.172 . This indicates minimal change in particle aggregative size after CUR loading in MNP formulation (ie, MNP formulation aggregative particle size was $116 \mathrm{~nm}$; Figure A1, Appendix). In addition, overall aggregative particles can be seen in low magnification TEM images which detect average aggregative size of nanoparticles $>120 \mathrm{~nm}$ (Figure A1B, Appendix). The average hydrodynamic particles size of MNP-CUR (123 nm) was considerably smaller than various multilayer iron oxide formulations prepared by coprecipitation approaches. ${ }^{31,40}$ The physical stability of a nanoparticle solution can be predicted by the zeta potential of the formulation. The zeta potential of the MNP-CUR formulation was $-0.66 \mathrm{mV}$ which allows good
A
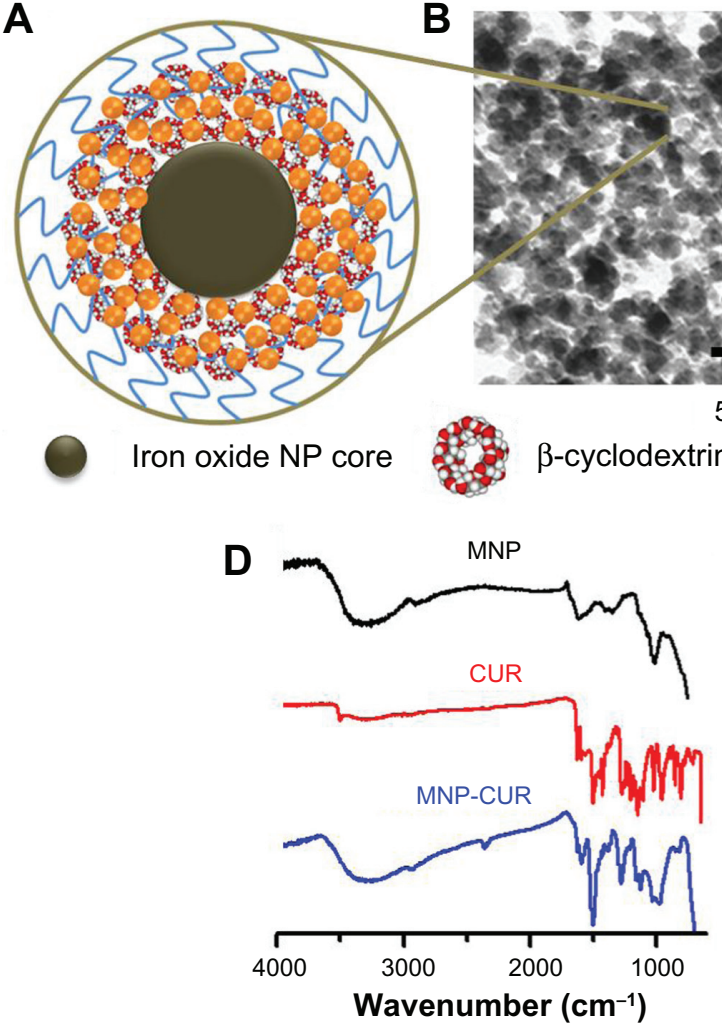

C

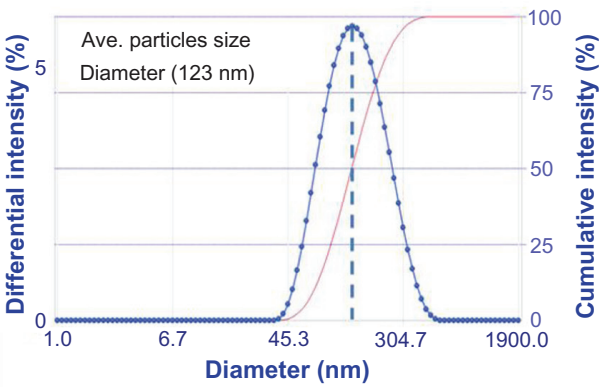

F-68 polymer chain

Curcumin
E

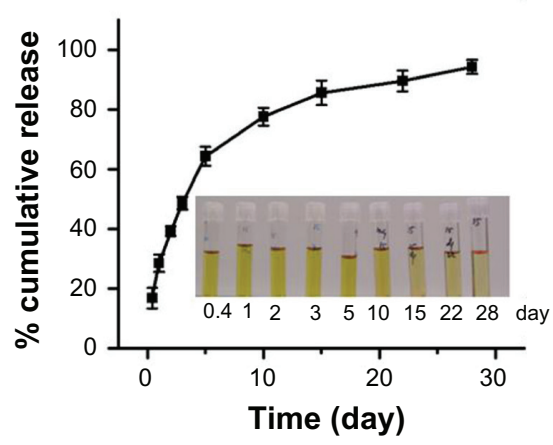

Figure I Physicochemical characterization of curcumin-loaded magnetic nanoparticle formulation. (A) Hypothetical schematic representation of curcumin-loaded single magnetic nanoparticle formulation. (B) Transmission electron microscopic image of curcumin-loaded magnetic nanoparticle formulation. Data show an individual particle grain size of $\sim 9 \mathrm{~nm}$. (C) Dynamic light scattering measurement of curcumin-loaded magnetic nanoparticle formulation. Data demonstrates the average nanoparticle aggregative size is $123 \mathrm{~nm}$. Data represents the average of three replicates. (D) Fourier transform infrared spectra of magnetic nanoparticle formulation, curcumin, and curcumin-loaded magnetic nanoparticle formulation. Data was obtained for dry powder samples. (E) In vitro curcumin release from curcumin-loaded magnetic nanoparticle formulation. Inset tubes depict curcumin release at different time points (0.4-28 days). Data represents the mean \pm standard error of the mean for three repeats.

Abbreviations: CUR, curcumin; MNP, magnetic nanoparticle formulation; MNP-CUR, curcumin-loaded magnetic nanoparticle formulation; NP, nanoparticle. 
A

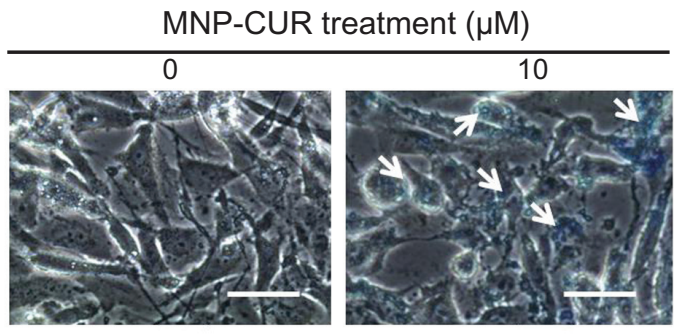

20 30
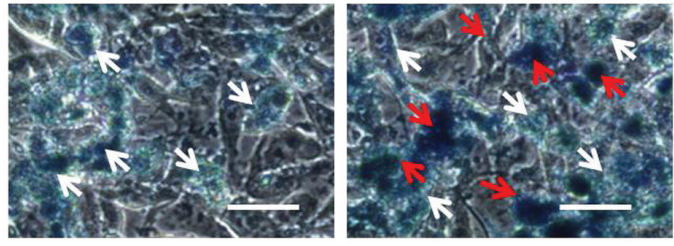

$\overline{20 \mu \mathrm{m}}$

Control

B MDA-MB-231 cancer cells
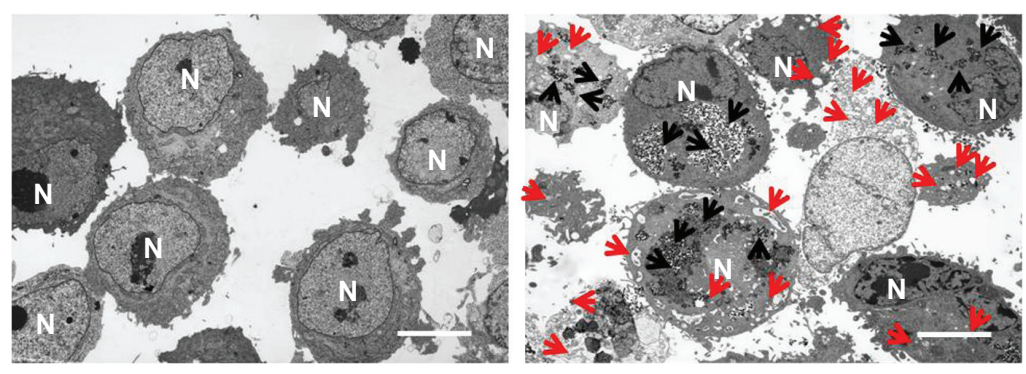

MNP-CUR treated MDA-MB-231 cancer cells

$2 \mu \mathrm{m}$

Figure 2 Curcumin-loaded magnetic nanoparticle formulation improves curcumin cellular uptake. (A) Phase contrast images of MDA-MB-23I cancer cells treated with curcumin-loaded magnetic nanoparticle formulation (0-30 $\mu \mathrm{M})$. The intensity of blue stain produced by Prussian blue from the iron of curcumin-loaded magnetic nanoparticle formulation helps improve visualization of internalized nanoparticles of curcumin-loaded magnetic nanoparticle formulation. White arrows highlight less internalization of nanoparticles and red arrows indicate greater accumulation of nanoparticles. (B) Transmission electron microscopic images of free MDA-MB-23 I cells and MDA-MB-23I cells incubated with curcumin-loaded magnetic nanoparticle formulation $(20 \mu \mathrm{M})$ for 6 hours. Black arrows indicate the accumulation and internalized nanoparticles (curcuminloaded magnetic nanoparticle formulation) and red arrows represent the vacuole and lysosomal activity which induces apoptosis.

Abbreviations: MNP-CUR, curcumin-loaded magnetic nanoparticle formulation; $\mathrm{N}$, nucleus.

stability during storage. The slightly negative zeta potential was attributed to the F68 polymer. Because the zeta potential for nanoparticles in solution is a slightly negative value, the nanoparticles may likely repel each other resulting in less aggregation. A similar observation was found in the case of oleic acid-F127 pluronic polymer-stabilized MNPs. ${ }^{41}$

The chemical difference between MNP and MNP-CUR formulations was evaluated by FTIR analysis (Figure 1D). FTIR of MNPs (black color spectrum) clearly showed a broad peak between $3600-3050 \mathrm{~cm}^{-1}$ due to the presence of hydroxyl groups of iron oxide core/ $\mathrm{CD} / \mathrm{F} 68$ polymer, and an intense band at $1010 \mathrm{~cm}^{-1}$ due to glycosidic $\mathrm{C}-\mathrm{O}-\mathrm{C}$ vibration of CD and F68 polymer. ${ }^{21,32,40,42}$ FTIR analysis of CUR (red color) revealed peaks at: $1610 \mathrm{~cm}^{-1}$ and $1500 \mathrm{~cm}^{-1}$ due to $\mathrm{C}=\mathrm{C}$ and $\mathrm{C}=\mathrm{O}$ of benzene ring, respectively, $1438 \mathrm{~cm}^{-1}$ due to olefinic $\mathrm{C}-\mathrm{H}$ bending vibration, $1280 \mathrm{~cm}^{-1}$ due to aromatic $\mathrm{C}-\mathrm{O}$ stretching vibrations, and $985 \mathrm{~cm}^{-1}$ due to $\mathrm{C}-\mathrm{O}-\mathrm{C}$ stretching vibrations. FTIR spectrum of the MNP-CUR formulation demonstrated peaks related to CUR, in addition to MNP related peaks, that are consistent with the authors' previous reports (on poly[lactic-co-glycolic acid]-CUR nanoparticles and CD-CUR nanoassemblies) and published literature. ${ }^{19,21,43,44}$

The loading efficiency of therapeutic drugs in nanoparticles directly influences the therapeutic outcome of the system. CUR is a known anticancer and chemopreventive molecule. ${ }^{8}$ However, its use is limited due to its low bioavailability and poor pharmacokinetics. Therefore, a MNP-CUR nanosystem was developed to increase the bioavailability, pharmacokinetics and subsequently improve the therapeutic efficacy of CUR. The dual layer MNP formulation permitted loading of $94.2 \mu \mathrm{g}$ of CUR per mg of nanoparticles. This level of drug loading was likely achieved because the presence of a CD layer shell at the interface of the MNP 
core and F68 polymer chains may prevent the diffusion of CUR. ${ }^{21,45,46}$ Figure 1E demonstrates the release profile of CUR from MNP-CUR under in vitro conditions. A slow and sustained release of CUR from MNP-CUR was noticed throughout the study (for 28 days). About 47\% CUR release occurred in the first 5 days. Therefore, this data clearly supports the proposition that CUR release is highly controlled by the CD layer. Since CUR is a hydrophobic molecule, it therefore remains in the $\mathrm{CD}$ layers (hydrophobic cavity) rather than transfer to the aqueous phase. Additionally, the F68 polymer chains (polypropylene oxide units) may also restrict the release of CUR into aqueous medium. Therefore, the MNP-CUR formulation exhibited controlled and sustained release of CUR which may ensure its efficient anticancer activity and prevent the cancer from developing drug resistance. ${ }^{47}$

\section{Cellular uptake}

Improved intracellular accumulation or uptake of drug nanoparticles in cancer cells is an indication for a higher therapeutic index. ${ }^{16}$ Therefore, this step of the present study investigated the qualitative uptake of MNP-CUR by MDA-MB-231 cells. The uptake of MNP-CUR was visualized by Prussian blue staining. A clear accumulation pattern of MNP-CUR was observed in MDA-MB-231 cancer cells: accumulation increases as the MNP-CUR concentration increases (faint blue [white arrows] to dark blue [red arrows]; Figure 2A). The internalized particles localized in almost every cell and throughout the cell components. This type of formulation showed very minimal uptake by macrophages, which supports increased circulation time for a more effective therapy. ${ }^{22,41}$ It is also logically possible to improve the internalization capacity of this type of nanoparticle by a ligand/antibody/penetrating peptide. ${ }^{48}$ Their high affinity allows preferential internalization through receptor mediated endocytosis; in this manner nanoparticle(s) efficiently target cancer cells.

To further prove MNP-CUR intracellular localization in MDA-MB-231 cancer cells, a TEM study was performed. In this study, after 6 hours treatment with MNP-CUR, MDA-MB-231 cancer cells were cross-sectioned (90 nm) using an ultramicrotome and viewed under TEM according to a previously reported method. ${ }^{19}$ Interestingly, most of the MNP-CURs were located as clusters in the cell structure, a phenomenon which was completely absent in control cells (Figure 2B). A magnified view of MNP-CUR uptake is also presented in Figure A2, Appendix. Therefore, it is clear that MNP-CURs underwent endocytosis but were not physically bound to the cancer cell surface. The authors propose that the entrapped MNP-CUR will demonstrate its anticancer properties by delivering CUR more efficiently to cancer cells. Control cancer cells were not affected at ultrastructural level and did not show any changes in cellular integrity. However, it is very clear that cancer cells treated with MNP-CUR exhibit ultra-structural changes, including shrinkage in nucleus, vesicle, and vacuole formation which represent onset of apoptosis. ${ }^{19,49}$

\section{Protein binding}

The final aim is to develop MNP-CUR for preclinical and human applications. Serum protein quickly binds to the surface of nanoparticles and changes their fate before reaching the intended target(s). This binding affinity of protein may depend on particle size and surface properties of the nanoparticles. ${ }^{49}$ Properties (in this case particle size) of stable drug nanoformulations may not be affected by the protein absorption during circulation in blood. To check this phenomenon, MNP-CUR formulation was incubated with human and mouse serum, and their particle size was measured using TEM and dynamic light scattering. Figure $3 \mathrm{~A}$ demonstrates no significant change in the aggregation of MNP-CUR formulation before and after incubation with human serum and mouse serum. However, there was a slight change in the surface morphology when incubated with mouse or human serum. Similarly, a slight increase in particle size was observed after incubation with mouse or human serum (MNP-CUR-mouse serum, $136 \mathrm{~nm}$; MNP-CUR-human serum, $144 \mathrm{~nm}$ ) compared to control nanoparticles (MNP-CUR, $123 \mathrm{~nm}$ ) (Figure 3B). The increase in particle size may be attributed to the adsorbed layers of serum proteins. ${ }^{50}$

\section{Anticancer potential}

It is imperative to assess the cytotoxicity of developed MNP-CUR for its potential anticancer application(s). The evaluation of cytotoxic activity was performed by direct observation of cell morphology as well as MTS assay. Evaluation by two different methods was conducted because some recent reports suggest that cytotoxicity assay reagents may interfere with nanoformulations. ${ }^{51,52}$ Cell image/morphology evaluation (Figure 4A) and MTS assays (Figure 4B) provide a direct qualitative and quantitative assessment of the anticancer potential of CUR/MNP-CUR formulations. When cells were treated with CUR, the viability of the cancer cells decreased sharply in a concentration (10-30 $\mu \mathrm{M}$ )-dependent manner (Figure 4A). A similar pattern was observed with MNP-CUR at all tested concentrations (Figure 4A). Interestingly, cell morphology was drastically 


\section{A} MNP-CUR

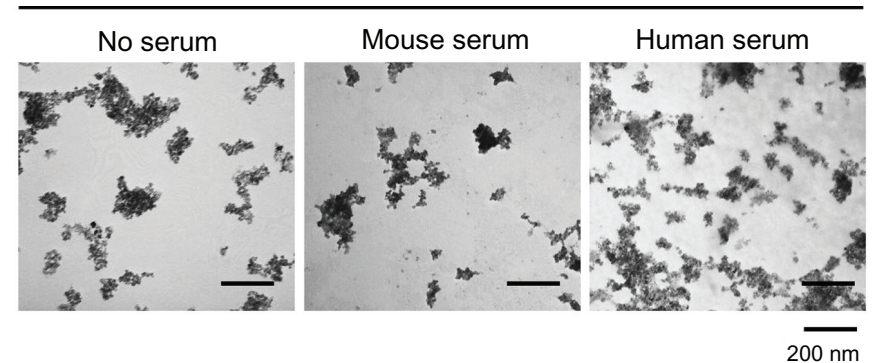

B

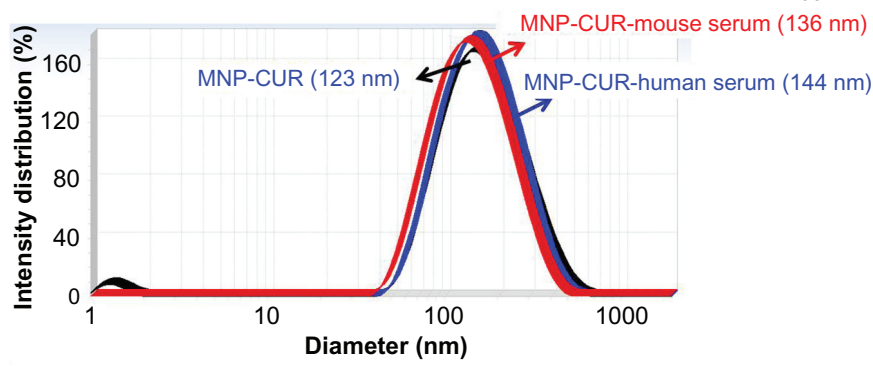

Figure 3 Curcumin-loaded magnetic nanoparticle formulation particle size is not influenced by human and mouse serum protein adsorption. (A) Curcumin-loaded magnetic nanoparticle formulation particles morphology viewed under transmission electron microscope before and after incubation with human and mouse serum. (B) Particle size of curcumin-loaded magnetic nanoparticle formulation before and after incubation with human and mouse serum.

Abbreviation: MNP-CUR, curcumin-loaded magnetic nanoparticle formulation.
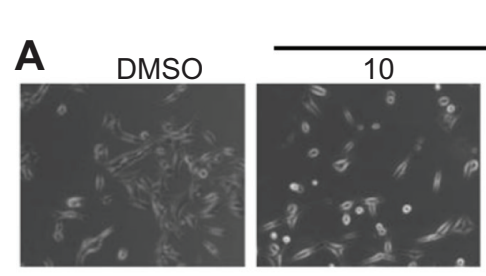

$\operatorname{CUR}(\mu \mathrm{M})$
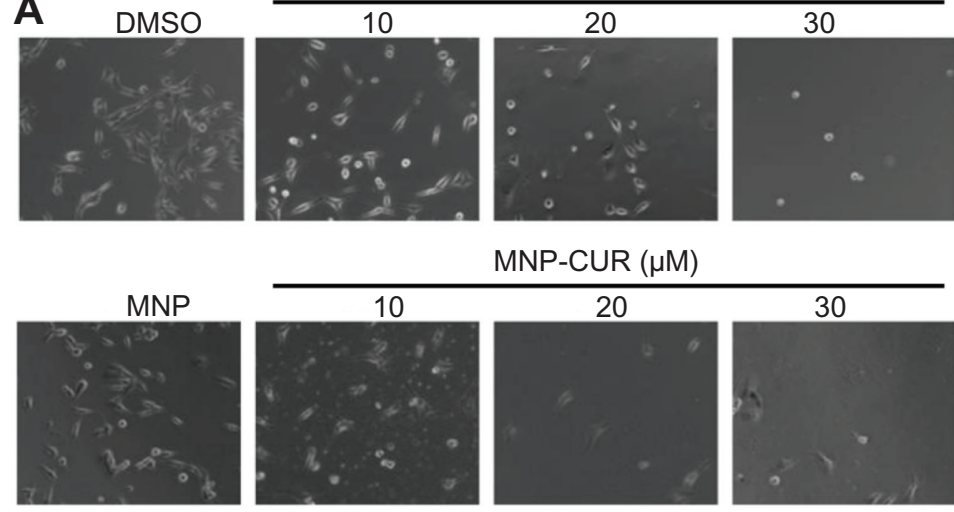

MNP-CUR $(\mu \mathrm{M})$
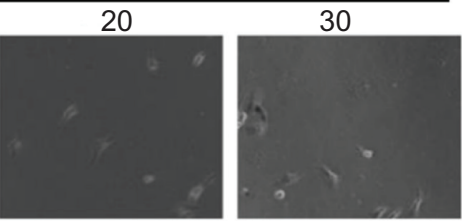

C
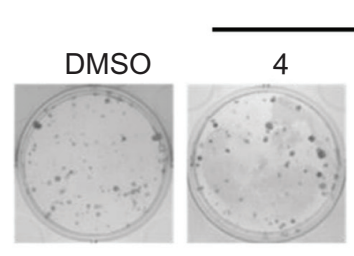

CUR $(\mu \mathrm{M})$

6

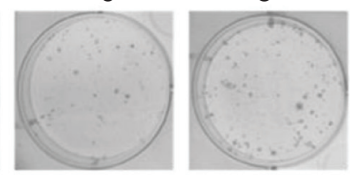

MNP-CUR $(\mu \mathrm{M})$

MNP

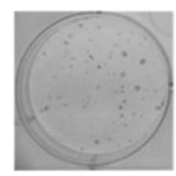

4

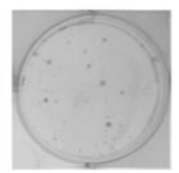

6

8
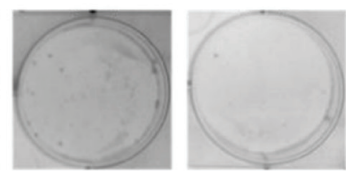

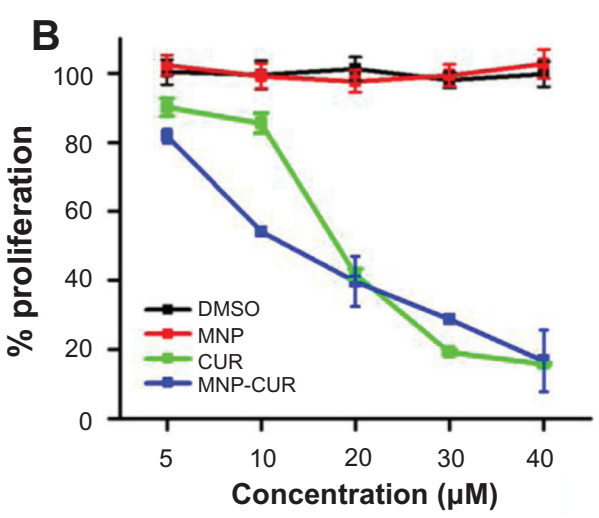

D

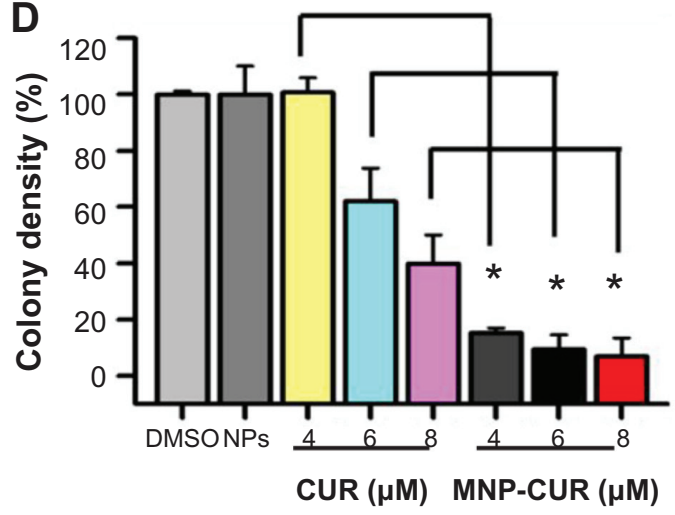

Figure 4 Curcumin-loaded magnetic nanoparticle formulation treatment increases the anticancer potential in MDA-MB-23I cells. (A and B) Cells were treated with different concentrations $(5-40 \mu \mathrm{M})$ of curcumin or curcumin-loaded magnetic nanoparticle formulation; medium was changed on day two. (A) Representative phase contrast images of MDAMB-23I cells treated with vehicle (dimethyl sulfoxide or magnetic nanoparticles) and 10-30 $\mu$ M curcumin or I0-30 $\mu$ M curcumin-loaded magnetic nanoparticle formulation. Images obtained at 2000x. (B) Cell viability was measured using methyl tetrazolium salt assay using an ultraviolet-visible spectrophotometer at $492 \mathrm{~nm}$. Data is mean \pm standard error of the mean $(n=6)$. Dimethyl sulfoxide and magnetic nanoparticle formulation controls did not show any effect at these concentrations. (C and $\mathbf{D})$ Cells were treated with either 4-8 $\mu$ M of curcumin or curcumin-loaded magnetic nanoparticle formulation for 10 days. (C) Representative images of colonies stained with hematoxylin. (D) Colony density was counted and expressed as a percent of the control. Data represent mean of triplicate for each treatment (mean \pm standard error of the mean). $* P<0.05$ for significance difference. Abbreviations: CUR, curcumin; DMSO, dimethyl sulfoxide; MNP, magnetic nanoparticle formulation; MNP-CUR, curcumin-loaded magnetic nanoparticle formulation; NPs, nanoparticles. 
changed and the number of cancer cells was greatly reduced even at $10 \mu \mathrm{M}$ MNP-CUR. A very limited number of cancer cells were observed after treatment with $20 \mu \mathrm{M}$ and $30 \mu \mathrm{M}$ of CUR/MNP-CUR. CUR/MNP-CUR-treated cells were clearly distinguished with plasma membrane smoothing, blebbing, and loss of spindle morphology, and were almost detached, which is an apoptosis feature. Treatments with various CUR and CUR nanoformulations also have reported this apoptosis feature in the number of cancer cells. ${ }^{16,23}$ The MTS assay data demonstrated a concentration-dependent antiproliferative effect $(5-40 \mu \mathrm{M})$ by CUR and MNP-CUR formulations (Figure 4B) as observed in the study of cellular images. The half maximal inhibitory concentration $(50 \%$ cell growth inhibitory concentration) was calculated as $17.2 \mu \mathrm{M}$ with CUR and $12.4 \mu \mathrm{M}$ with MNP-CUR treatment. From this data, it can be concluded that although CUR release from MNP-CUR formulation was only $40 \%$, it is as equally potent as free CUR in suppressing cell growth. Therefore, the MNP-CUR formulation can be employed as an efficient cancer therapeutic agent.

To further prove that the MNP-CUR formulation holds improved anticancer therapeutic potential, a propidium iodide stain experiment was performed. Propidium iodide specifically binds to double-stranded DNA through the plasma membrane of nonviable cells which can be detected in flow cytometry in the FL2 channel. MNP-CUR-treated cells indicate a significant propidium iodide stain over CURtreated cells, suggesting a higher number of dead cells exist after MNP-CUR treatment (Figure A3, Appendix). This data supports the findings of MTS assay.

The authors' hypothesis is that since CUR releases slowly from MNP-CUR, this formulation may exhibit a superior anticancer activity in long term experiments such as colony formation assays. For example, magnetoplasmonic assembly of the polyethylene glycol-CUR formulation demonstrates better anticancer properties after 42-52 hours treatment compared to magnetoplasmonic assembly-CUR/ CUR treatment and the efficacy depends on the drug release pattern. ${ }^{49}$ This supports the belief that CUR nanoformulations require time to show superior effects. Therefore, the efficacy of MNP-CUR on MDA-MB-231 cancer cells was studied. A significant decrease was observed in the formation of colonies after MNP-CUR treatment compared to CUR (Figure 4C). CUR treatment gradually declined formation of colony density, ie, 4-8 $\mu \mathrm{M}$ concentration was responsible for reducing colony density from $\sim 100 \%$ to $38 \%$ (Figure 4D) whereas $4 \mu \mathrm{M}$ MNP-CUR treatment successfully restricted colony density to merely $16 \%$ (Figure 4D). This result clearly indicates that the increased cytotoxicity (reduced colony density) was primarily associated with active CUR release from MNP-CUR over time. A similar improved anticancer effect in a number of cancer cell lines was observed in previous studies using various CUR formulations. ${ }^{18-20}$ These studies support this enhanced anticancer potential by documenting an increase in apoptosis as demonstrated through enhanced cleaved poly(adenosine diphosphate ribose) polymerase protein with MNP or other CUR nanoformulations. ${ }^{18-20}$

\section{Mitochondrial membrane potential}

The loss of mitochondrial membrane or mitochondrial dysfunction is a key indicator of intrinsic apoptosis. ${ }^{42}$ The specificity of mitochondrial stain after 24 hours treatment with CUR and MNP-CUR formulations was detected using cationic dye TMRE by fluorescence microscopy and flow cytometry. TMRE accumulates in the mitochondria when a membrane has intact potential. TMRE positive cells are an indication of viable cells with high membrane potential. Decrease in TMRE stain indicates a loss of mitochondrial membrane potential. Microscopy analysis illustrated that upon treatment with $5 \mu \mathrm{M}$ CUR, the cells had a very similar amount of stain as control cells; however, after treatment with $10 \mu \mathrm{M}$ CUR, the cells showed a significant decrease in stain intensity (Figure 5A). In the case of MNP-CUR, both $5 \mu \mathrm{M}$ and $10 \mu \mathrm{M}$ treatments showed decreased stain, indicating effective mitochondrial membrane damage and thus increased anticancer potential (Figure 5A). Similar results were observed with flow cytometry analysis. A significant decrease in TMRE stain was noticed with MNP-CUR treatment compared to CUR (Figure 5B). This may be due to higher cellular uptake of MNP-CUR, inducing greater loss in membrane potential.

\section{ROS production}

Many anticancer drug treatments lead to the generation of ROS which is involved in stimulating inflammation, apoptosis, and DNA damage which can affect the signaling pathways involved in external or internal cell damage. ${ }^{53,54}$ Although CUR is a potent antioxidant with ROS scavenger characteristics, it also induces ROS production in human tumor cells. ${ }^{55}$ A MitoSOX stain was used to identify such intracellular mitochondrial ROS generation from the CUR-treated and MNP-CUR-treated cells. ROS production in the CURtreated cells was not significantly different from control cells. However, the production of ROS was significantly increased 
A
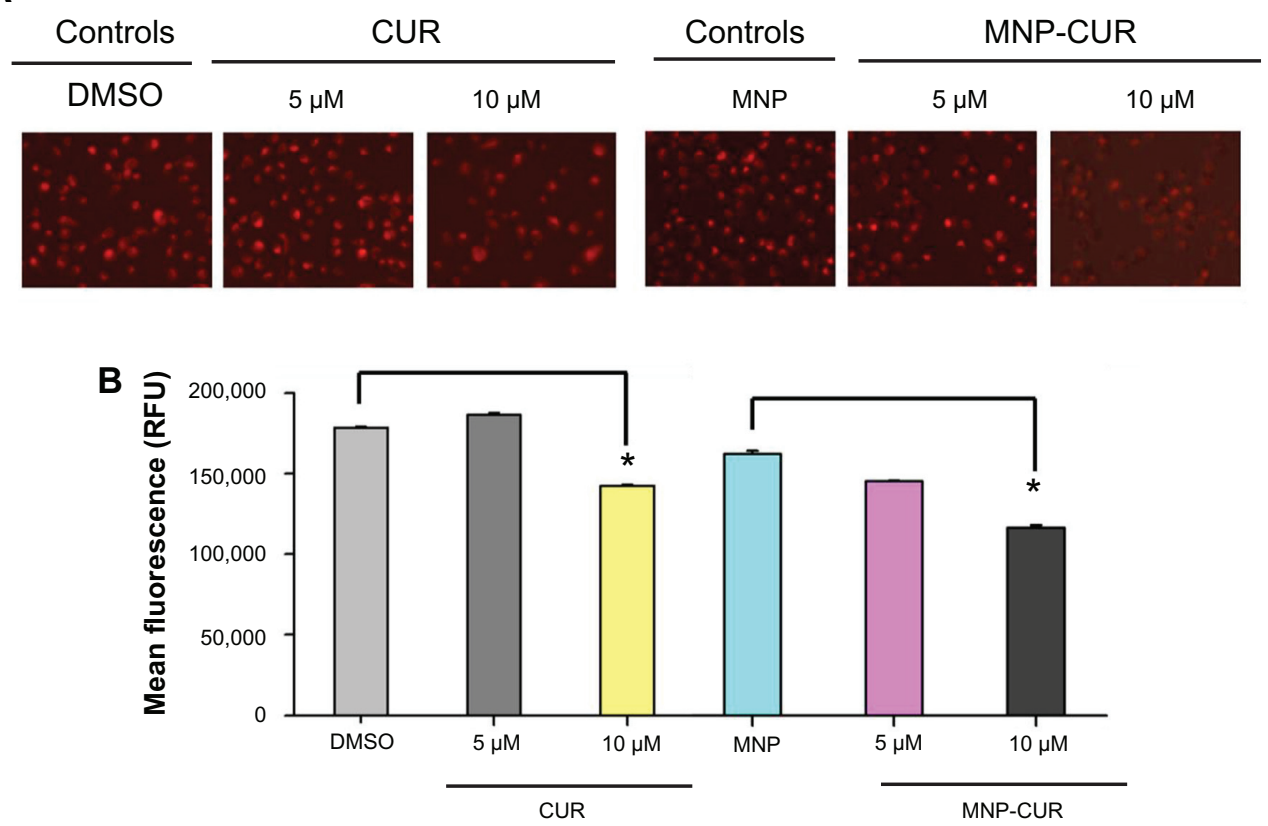

Figure 5 Curcumin-loaded magnetic nanoparticle increases loss of mitochondrial membrane potential. MDA-MB-23I cells $\left(I \times 10^{6}\right)$ were treated with curcumin and curcumin-loaded magnetic nanoparticles $(0-10 \mu \mathrm{M})$ for 24 hours and the loss of mitochondrial membrane potential was determined using tetramethyl rhodamine ethyl ester dye. (A) Representative tetramethyl rhodamine ethyl ester stain in MDA-MB-23I cells after treatment with curcumin and curcumin-loaded magnetic nanoparticle formulation. Less intense stain indicates loss of mitochondrial membrane potential. (B) Quantitative estimation of tetramethyl rhodamine ethyl ester stain was analyzed by flow cytometry. For this analysis, 10,000 cells were injected into an Accuri ${ }^{\circledR}$ C6 Flow Cytometer (BD Accuri Cytometers, Ann Arbor, MI) and fluorescence levels were measured in the FL2 channel. Data represent the mean of three repeats for each treatment (mean \pm standard error of the mean). $* P<0.05$ for significance difference.

Abbreviations: CUR, curcumin; DMSO, dimethyl sulfoxide; MNP, magnetic nanoparticle formulation; MNP-CUR, curcumin-loaded magnetic nanoparticle formulation; RFU, relative fluorescence units.

in MNP-CUR-treated cells (Figure 6A and B). These results further demonstrate that MNP-CUR can efficiently internalize and deliver CUR in its active form and is responsible for ROS production, while free CUR barely influences the production of ROS at the tested concentrations. The obtained results corroborate similar findings reported by Mulik et $\mathrm{al}^{56}$ that CUR nanoparticles potentiate apoptosis in MDA-MB-231 cancer cells via generation of ROS. Similarly, pretreatment and cotreatment of CUR nanoparticles with other anticancer drugs also leads to an increase in ROS production. ${ }^{57,58}$

\section{MRI}

MNPs exhibit specific inherent magnetic properties that are highly important in MRI for increasing the detection limits in tissue lesions within the body. ${ }^{24,25,38}$ In addition, surfacemodified MNPs avoid recognition by the reticuloendothelial system and therefore may efficiently target tumors, making them a better contrast imaging agent. In this study, the suitability of MNPs and the MNP-CUR formulation as contrast imaging agents for MRI was investigated. ${ }^{26,38}$ Figure 7A illustrates an increase of nanoparticle concentration resulting in a reduction in $\mathrm{T}_{2}$-weighted signals. Calculated transverse relaxation times from images (Figure 7A) relative to the control gel decreased with an increase in nanoparticle concentration (Figure 7B). Specifically, as the concentration increased from $10 \mu \mathrm{g}$ iron $/ \mathrm{mL}$ to $40 \mu \mathrm{g}$ iron $/ \mathrm{mL}, \mathrm{T}_{2}$ relaxation times decreased from 22.4 milliseconds to 5.8 milliseconds. By plotting the transverse relaxation rate $R_{2}\left(1 / T_{2}\right)$ as a function of the concentration of iron in each sample, $\mathrm{R}_{2}$ increased linearly with the concentration of iron (Figure $7 \mathrm{C}$ ). From these data, $T_{2}$ relaxivity $\left(r_{2}\right)$ was calculated as $7.040 \mathrm{~s}^{-1} \mu \mathrm{g}^{-1} \mathrm{~mL}$ for MNP-CUR. The relaxivity was slightly diminished when the particles were in the presence of cancer cells $\left(4.38 \mathrm{~s}^{-1} \mu \mathrm{g}^{-1} \mathrm{~mL}\right)$ but enhanced values were observed in combination with CURnanoparticles. The relaxivity order detected was $7.040 \mathrm{~s}^{-1} \mu \mathrm{g}^{-1}$ $\mathrm{mL}(\mathrm{MNP}-\mathrm{CUR})>4.38 \mathrm{~s}^{-1} \mu \mathrm{g}^{-1} \mathrm{~mL}(\mathrm{MNP}-\mathrm{CUR}$ in cancer cells $)>3.40 \mathrm{~s}^{-1} \mu \mathrm{g}^{-1} \mathrm{~mL}(\mathrm{MNP})>2.38 \mathrm{~s}^{-1} \mu \mathrm{g}^{-1} \mathrm{~mL}(\mathrm{MNP}$ in cancer cells). A similar pattern was also observed in $\mathrm{T}_{1}$ relaxivity $\left(\mathrm{r}_{1}\right): 33.21 \mathrm{~s}^{-1} \mu \mathrm{g}^{-1} \mathrm{~mL}(\mathrm{MNP}-\mathrm{CUR})>18.663 \mathrm{~s}^{-1} \mu \mathrm{g}^{-1} \mathrm{~mL}$ $($ MNP-CUR in cells $)>11.303 \mathrm{~s}^{-1} \mu \mathrm{g}^{-1} \mathrm{~mL}(\mathrm{MNP})>6.389 \mathrm{~s}^{-1}$ $\mu \mathrm{g}^{-1} \mathrm{~mL}$ (MNP in cancer cells). The higher relaxivity values of MNP-CUR are attributed to CUR and the nanoparticles induce greater inhomogeneity in the magnetic field than MNP. Similar improved enhanced contrast properties were observed 
A

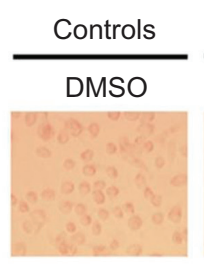

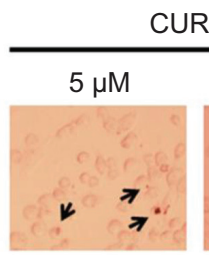

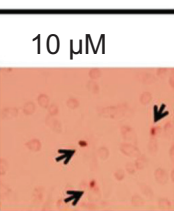

Controls

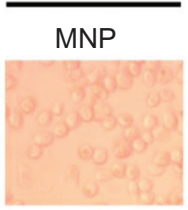

MNP-CUR

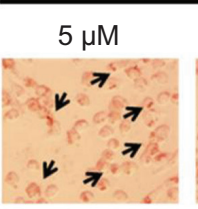

$10 \mu \mathrm{M}$

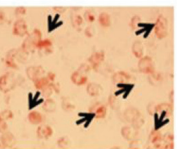

B

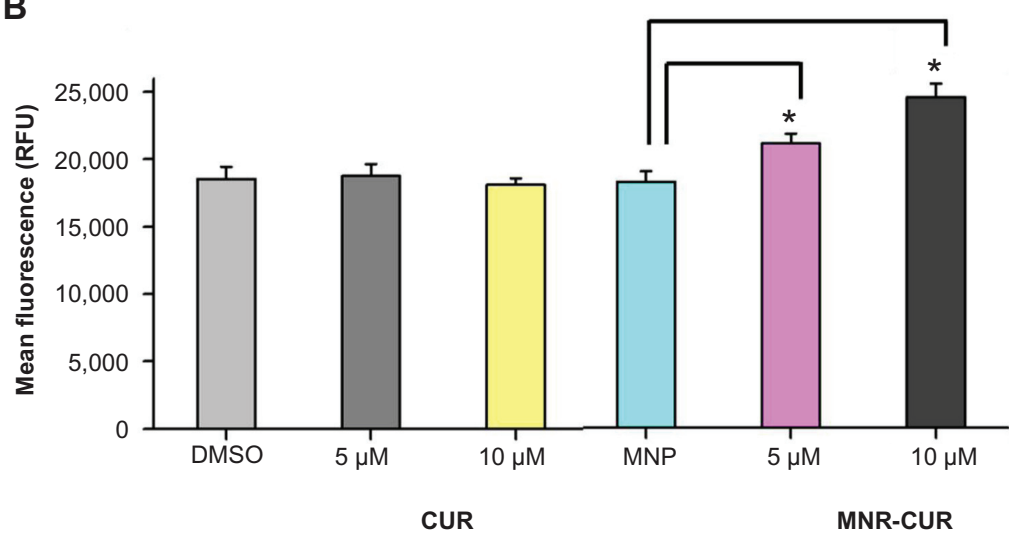

Figure 6 Curcumin-loaded magnetic nanoparticle formulation induces more reactive oxygen species formation. MDA-MB-23I cells $\left(I \times 10^{6}\right)$ were treated with curcumin or curcumin-loaded magnetic nanoparticle formulation (0-10 $\mu \mathrm{M}$ ) for 24 hours, and reactive oxygen species generation was determined using MitoSOX Red dye (Invitrogen Life Technologies, Carlsbad, CA). (A) Representative reactive oxygen species generation images in MDA-MB-23I cells after treatment with curcumin and curcumin-loaded magnetic nanoparticle formulation. (B) Quantitative estimation of reactive oxygen species generation was analyzed by flow cytometer. For this analysis, 10,000 cells were injected into an Accuri ${ }^{\circledR} \mathrm{C} 6$ flow cytometer (BD Accuri Cytometers, Ann Arbor, MI) and fluorescence levels were measured in the FL2 channel. Data represent the mean of three repeats for each treatment (mean \pm standard error of the mean). $* P<0.05$ for significance difference.

Abbreviations: CUR, curcumin; DMSO, dimethyl sulfoxide; MNP, magnetic nanoparticle formulation; MNP-CUR, curcumin-loaded magnetic nanoparticle formulation; RFU, relative fluorescence units.
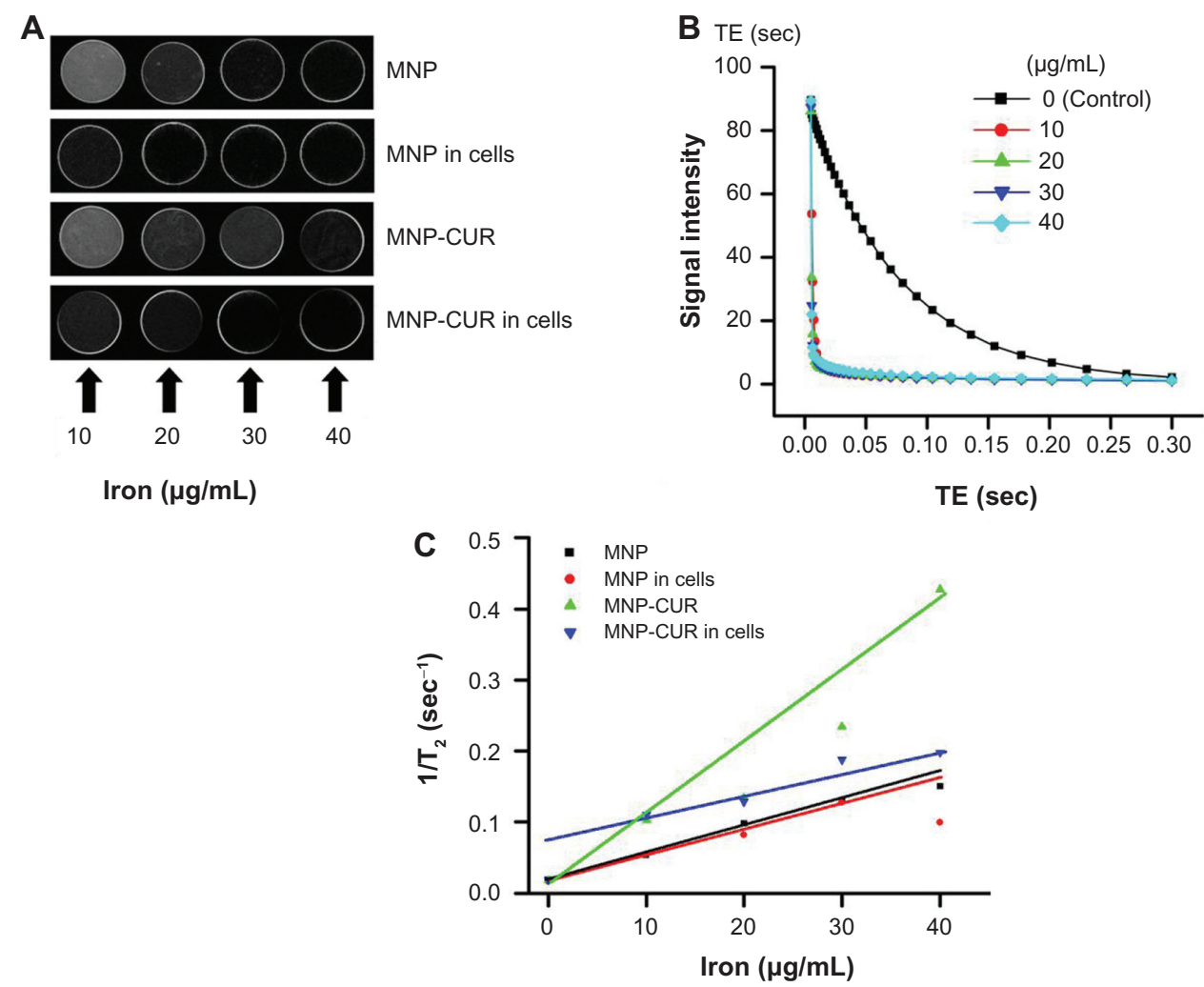

Figure 7 In vitro magnetic resonance image characteristics of magnetic nanoformulations. (A) Signal intensity $T_{2}$-weighted magnetic resonance images of magnetic nanoparticle formulation, magnetic nanoparticle formulation in MDA-MB-23I cancer cells, curcumin-loaded magnetic nanoparticle formulation, and curcumin-loaded magnetic nanoparticle formulation in MDA-MB-23I cancer cells in 3\% phantom agar gel (10-40 $\mu \mathrm{g} / \mathrm{mL})$ scanned under a $9.4 \mathrm{~T}$ vertical bore magnetic resonance image scanner (Agilent Technologies, Santa Clara, CA) at $25^{\circ} \mathrm{C}$. (B) $T_{2}$ relaxation curves of magnetic nanoformulations in phantom agar gel. (C) $T_{2}$ relaxation rate $R_{2}\left(I / T_{2}\right)$ plotted as a function of the iron concentration for magnetic nanoformulations.

Abbreviations: MNP, magnetic nanoparticle formulation; MNP-CUR, curcumin-loaded magnetic nanoparticle formulation; TE, echo time. 
with drug-loaded formulations due to the color(s) contributed by the drug(s), such as yellow (CUR) or red (doxorubicin). ${ }^{21,31,39}$ Previous reports support that these multilayer-coated MNPs may have superior imaging characteristics over conventional magnetic nanoformulations. ${ }^{40,41}$

\section{Magnetic targeting}

Ideal magnetic field mediated drug delivery (magnetic targeted drug delivery) facilitates drug accumulation in cells possibly by passive diffusion, adsorptive transcytosis, and receptor mediation. ${ }^{59}$ This advanced drug targeting approach is increasingly utilized due to increased site specificity with fewer side effects. ${ }^{26}$ However, efficiency of magnetic field-mediated cellular delivery of drug depends on the applied external magnetic field as well as the type of MNP carrier. ${ }^{25,38,60,61}$ To determine the potential utility of the nanoformulations for possible magnetic targeting, MDA-MB-231 cancer cells treated with MNP-CUR were exposed to a neodymium external magnet for 3 hours. A detailed schematic representation of the experiment design is presented in Figure 8A. The results were evaluated comparing CUR/MNP-CUR internalization in the cancer cells. CUR (2.5-7.5 $\mu \mathrm{M})$ exposed cells exhibited a very minute amount of CUR. MNP-CUR-treated cells demonstrated significant cellular uptake of CUR through the influence of magnetic field (Figure 8B). This effect was only minimal when there was no magnetic field applied (Figure 8B). Overall, qualitative accumulation/cellular uptake of CUR assessed by flow cytometry was clearly enhanced by magnetic field (Figure 8C) at all the concentrations tested. Thus, exposure to external magnets significantly increased the cellular uptake of MNPs predominantly into malignant cells. This study suggests that application of an external magnetic field would enhance additional CUR internalization, inducing more cellular apoptosis, thereby increasing the possible cytotoxicity of MNP-CUR.

Overall, the data show the feasibility of using an innovative nanoparticle system which combines magnetic targeting of a therapeutic agent (CUR) with both magnetic resonance and fluorescence imaging in a single system (Figure 9). This formulation can be extended to other clinically used therapeutic drugs and can be developed as antibody conjugation features. ${ }^{41}$ Targeting efficacy of these multifunctional nanoparticles specifically to triple-negative

\section{A CUR treatment}

MNP-CUR treatment
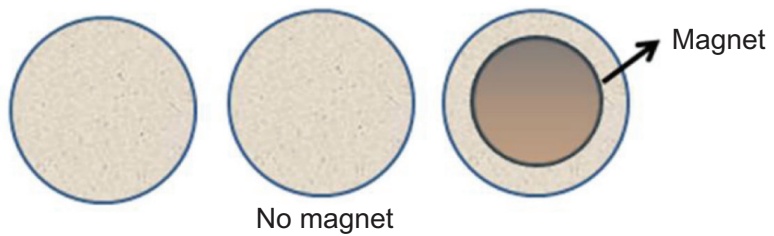

MNP-CUR
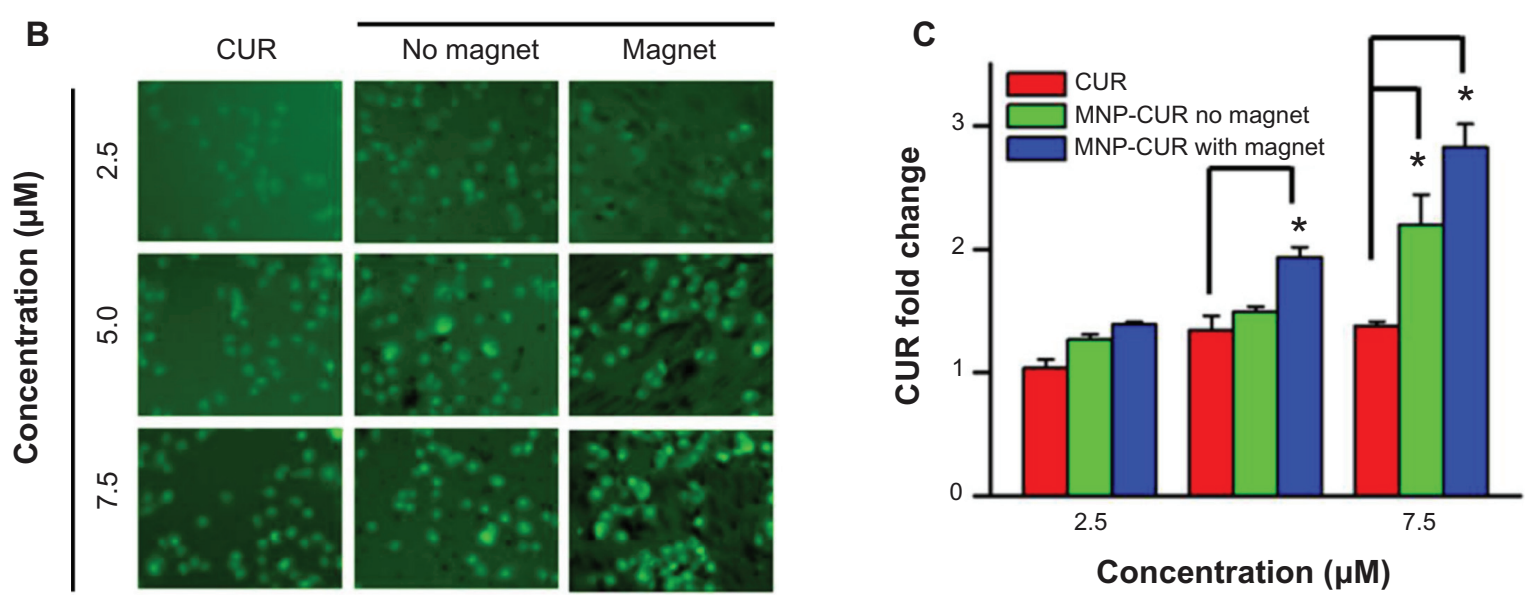

Figure 8 Curcumin-loaded magnetic nanoparticle formulation magnetic targeting improves curcumin uptake by MDA-MB-23। cells. (A) Schematic representation of experimental design for magnetic targeting. Cells $\left(2 \times 10^{5}\right)$ were seeded in six-well plates and treated for 3 hours with $2.5,5$, and $7.5 \mu$ M of $(I)$ curcumin, $(2)$ curcumin-loaded magnetic nanoparticle formulation, or (3) curcumin-loaded magnetic nanoparticle formulation in the presence of a neodymium external magnet. (B) Curcumin uptake by cancer cells was visualized using a fluorescence microscope (IX7I; Olympus Corporation, Tokyo, Japan). (C) Internalized fluorescence levels were analyzed using an Accuri ${ }^{\circledR}$ C6 flow cytometer (BD Accuri Cytometers, Ann Arbor, MI) in the FLI channel. Data represent the mean of three repeats for each treatment (mean \pm standard error of the mean) and expressed in fold change compared to respective controls. $* P<0.05$ for significance difference.

Abbreviations: CUR, curcumin; MNP-CUR, curcumin-loaded magnetic nanoparticle formulation. 

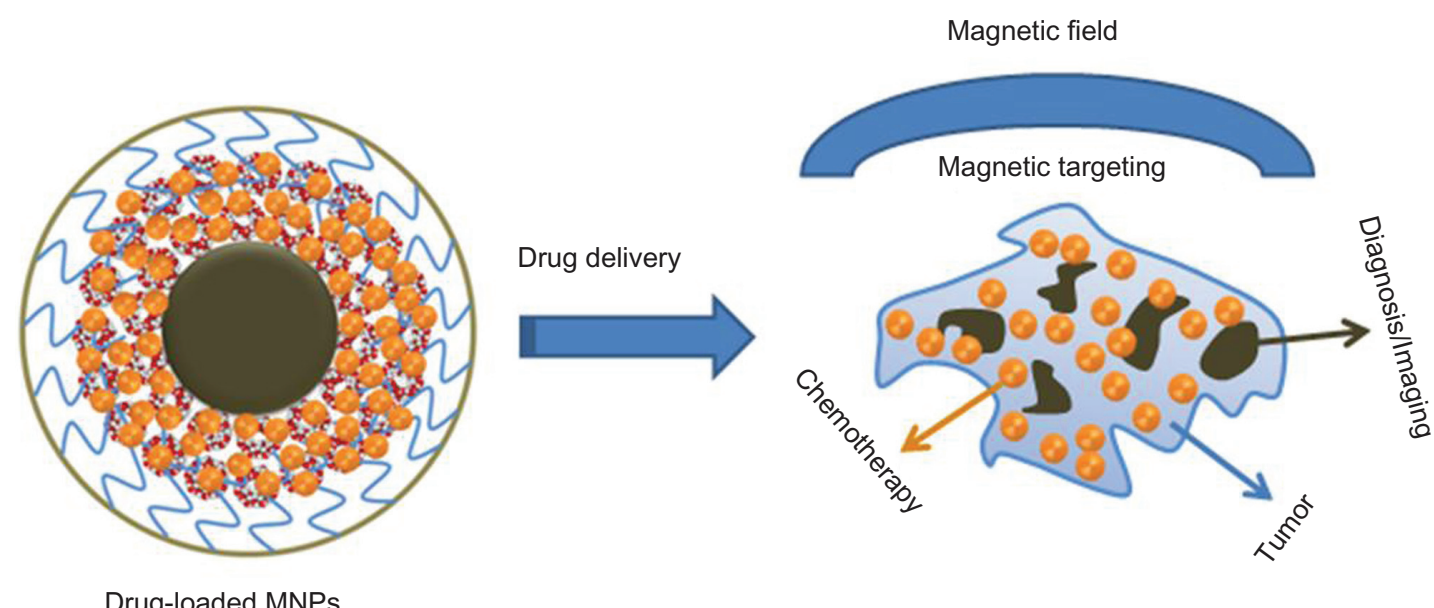

Figure 9 Schematic representation for theranostic application of MNP-CUR formulation.

Abbreviations: MNPs, magnetic nanoparticles; MNP-CUR, curcumin-loaded magnetic nanoparticle.

breast cancer cells can be further enhanced clinically through conjugation of monoclonal antibodies, such as antitransferrin (Figure A4, Appendix), glucose transporter 1, and folic acid. ${ }^{62,63}$

\section{Conclusion}

Development of safe, efficient, and biocompatible drug nanoformulations with theranostic properties is essential for clinical translation in cancer therapy. A composite MNP formulation composed of an iron oxide nanoparticle core coated with $\mathrm{CD}$ and pluronic polymer (F68) with CUR loading capability was developed. This formulation exhibited improved uptake in cancer cells, increased loss of mitochondrial membrane potential, and produced higher ROS, suggesting it is extremely valuable in anticancer therapeutics. Additionally, enhanced MRI properties (compared to conventional MNPs) demonstrate this formulation has good imaging agent characteristics. More importantly, magnetic targeting of MNP-CUR facilitated an enhanced delivery of CUR to cancer cells. All together, these findings indicate that this formulation is appropriate for utilization in engineered delivery systems for theranostic applications in efficient image-directed cancer therapeutics.

\section{Acknowledgments}

The authors thank Cathy Christopherson (Sanford Research/ University of South Dakota) for editorial assistance, Robert Japs (Sanford Research/University of South Dakota), and Sara Basiaga (University of Nebraska-Lincoln) for characterization of samples. This work was partially supported by grants from Department of Defense (PC073887, PC073643), Governor's Cancer 2010, the National Institutes of Health Research Project Grant Program (RO1) (CA142736), and the Centers of Biomedical Research Excellence (P20 RR024219).

\section{Disclosure}

The authors report no conflicts of interest in this work.

\section{References}

1. Siegel R, Ward E, Brawley O, Jemal A. Cancer statistics, 2011: the impact of eliminating socioeconomic and racial disparities on premature cancer deaths. CA Cancer J Clin. 2011;61(4):212-236.

2. Carey L, Winer E, Viale G, Cameron D, Gianni L. Triple-negative breast cancer: disease entity or title of convenience? Nat Rev Clin Oncol. 2010;7(12):683-692.

3. Carey LA. Triple-negative (basal-like) breast cancer: a new entity. Breast Cancer Res. 2007;9(Suppl 1):S13.

4. McGurk R, Fallowfield L, Winters Z. Information provision for patients by breast cancer teams about the side-effects of hormone treatments. Eur J Cancer. 2006;42(12):1760-1767.

5. Sjovall K, Strombeck G, Lofgren A, Bendahl PO, Gunnars B. Adjuvant radiotherapy of women with breast cancer - information, support and side-effects. Eur J Oncol Nurs. 2010;14(2):147-153.

6. Moore S. Managing treatment side effects in advanced breast cancer. Semin Oncol Nurs. 2007;23(4 Suppl 2):S23-S30.

7. Metzger Filho O, Saini KS, Azim HA Jr, Awada A. Prevention and management of major side effects of targeted agents in breast cancer. Crit Rev Oncol Hematol. March 15, 2011. [Epub ahead of print.]

8. Anand P, Sundaram C, Jhurani S, Kunnumakkara AB, Aggarwal BB. Curcumin and cancer: an "old-age" disease with an "age-old" solution. Cancer Lett. 2008;267(1):133-164.

9. Aggarwal BB, Banerjee S, Bharadwaj U, Sung B, Shishodia S, Sethi G. Curcumin induces the degradation of cyclin E expression through ubiquitin-dependent pathway and up-regulates cyclin-dependent kinase inhibitors p21 and p27 in multiple human tumor cell lines. Biochem Pharmacol. 2007;73(7):1024-1032.

10. Hutzen B, Friedman L, Sobo M, et al. Curcumin analogue GO-Y030 inhibits STAT3 activity and cell growth in breast and pancreatic carcinomas. Int J Oncol. 2009;35(4):867-872. 
11. Rowe DL, Ozbay T, O'Regan RM, Nahta R. Modulation of the BRCA1 protein and induction of apoptosis in triple negative breast cancer cell lines by the polyphenolic compound curcumin. Breast Cancer (Auckl). 2009;3:61-75.

12. Aggarwal BB, Shishodia S, Takada Y, et al. Curcumin suppresses the paclitaxel-induced nuclear factor-kappaB pathway in breast cancer cells and inhibits lung metastasis of human breast cancer in nude mice. Clin Cancer Res. 2005;11(20):7490-7498.

13. Goel A, Aggarwal BB. Curcumin, the golden spice from Indian saffron, is a chemosensitizer and radiosensitizer for tumors and chemoprotector and radioprotector for normal organs. Nutr Cancer. 2010;62(7):919-930.

14. Yallapu MM, Maher DM, Sundram V, Bell MC, Jaggi M, Chauhan SC. Curcumin induces chemo/radio-sensitization in ovarian cancer cells and curcumin nanoparticles inhibit ovarian cancer cell growth. J Ovarian Res. 2010;3:11.

15. Ravindran J, Prasad S, Aggarwal BB. Curcumin and cancer cells: how many ways can curry kill tumor cells selectively? AAPS J. 2009;11(3): 495-510.

16. Yallapu MM, Jaggi M, Chauhan SC. Curcumin nanoformulations: a future nanomedicine for cancer. Drug Discov Today. 2012;17(1-2) $71-80$.

17. Bansal SS, Goel M, Aqil F, Vadhanam MV, Gupta RC. Advanced drug delivery systems of curcumin for cancer chemoprevention. Cancer Prev Res (Phila). 2011;4(8):1158-1171.

18. Yallapu MM, Dobberpuhl MR, Maher DM, Jaggi M, Chauhan SC Design of curcumin loaded cellulose nanoparticles for prostate cancer. Curr Drug Metab. 2012;13(1):120-128.

19. Yallapu MM, Gupta BK, Jaggi M, Chauhan SC. Fabrication of curcumin encapsulated PLGA nanoparticles for improved therapeutic effects in metastatic cancer cells. J Colloid Interface Sci. 2010;351(1):19-29.

20. Yallapu MM, Jaggi M, Chauhan SC. Poly( $\beta$-cyclodextrin)/curcumin selfassembly: a novel approach to improve curcumin delivery and its therapeutic efficacy in prostate cancer cells. Macromol Biosci. 2010;10(10): 1141-1151.

21. Yallapu MM, Jaggi M, Chauhan SC. Beta-cyclodextrin-curcumin selfassembly enhances curcumin delivery in prostate cancer cells. Colloids Surf B Biointerfaces. 2010;79(1):113-125.

22. Yallapu MM, Othman SF, Curtis ET, Gupta BK, Jaggi M, Chauhan SC. Multi-functional magnetic nanoparticles for magnetic resonance imaging and cancer therapy. Biomaterials. 2011;32(7):1890-1905.

23. Anand P, Kunnumakkara AB, Newman RA, Aggarwal BB. Bioavailability of curcumin: problems and promises. Mol Pharm. 2007; 4(6):807-818.

24. Laurent S, Bridot JL, Elst LV, Muller RN. Magnetic iron oxide nanoparticles for biomedical applications. Future Med Chem. 2010;2(3): 427-449.

25. Shubayev VI, Pisanic TR II, Jin S. Magnetic nanoparticles for theragnostics. Adv Drug Deliv Rev. 2009;61(6):467-477.

26. Yoo D, Lee JH, Shin TH, Cheon J. Theranostic magnetic nanoparticles. Acc Chem Res. 2011;44(10):863-874.

27. Cole AJ, Yang VC, David AE. Cancer theranostics: the rise of targeted magnetic nanoparticles. Trends Biotechnol. 2011;29(7):323-332.

28. Ho D, Sun X, Sun S. Monodisperse magnetic nanoparticles for theranostic applications. Acc Chem Res. 2011;44(10):875-882.

29. Janib SM, Moses AS, MacKay JA. Imaging and drug delivery using theranostic nanoparticles. Adv Drug Deliv Rev. 2010;62(11): 1052-1063.

30. Tassa C, Shaw SY, Weissleder R. Dextran-coated iron oxide nanoparticles: a versatile platform for targeted molecular imaging, molecular diagnostics, and therapy. Acc Chem Res. 2011;44(10):842-852.

31. Jain TK, Morales MA, Sahoo SK, Leslie-Pelecky DL, Labhasetwar V. Iron oxide nanoparticles for sustained delivery of anticancer agents. Mol Pharm. 2005;2(3):194-205.

32. Jain TK, Richey J, Strand M, Leslie-Pelecky DL, Flask CA, Labhasetwar V. Magnetic nanoparticles with dual functional properties: drug delivery and magnetic resonance imaging. Biomaterials. 2008; 29(29):4012-4021.
33. Bhattarai SR, Kc RB, Kim SY, et al. N-hexanoyl chitosan stabilized magnetic nanoparticles: implication for cellular labeling and magnetic resonance imaging. J Nanobiotechnology. 2008;6:1.

34. Song M, Moon WK, Kim Y, Lim D, Song IC, Yoon BW. Labeling efficacy of superparamagnetic iron oxide nanoparticles to human neural stem cells: comparison of ferumoxides, monocrystalline iron oxide, cross-linked iron oxide(CLIO)- $\mathrm{NH}_{2}$ and tat-CLIO. Korean J Radiol. 2007;8(5):365-371.

35. Yallapu MM, Ebeling MC, Chauhan N, Jaggi M, Chauhan SC. Interaction of curcumin nanoformulations with human plasma proteins and erythrocytes. Int J Nanomedicine. 2011;6:2779-2790.

36. Gillis P, Koenig SH. Transverse relaxation of solvent protons induced by magnetized spheres: application to ferritin, erythrocytes, and magnetite. Magn Reson Med. 1987;5(4):323-345.

37. Gupta AK, Naregalkar RR, Vaidya VD, Gupta M. Recent advances on surface engineering of magnetic iron oxide nanoparticles and their biomedical applications. Nanomedicine (Lond). 2007;2(1): 23-39.

38. Namdeo M, Saxena S, Tankhiwale R, Bajpai M, Mohan YM, Bajpai SK. Magnetic nanoparticles for drug delivery applications. J Nanosci Nanotechnol. 2008;8(7):3247-3271.

39. Banerjee SS, Chen DH. Cyclodextrin conjugated magnetic colloidal nanoparticles as a nanocarrier for targeted anticancer drug delivery. Nanotechnology. 2008;19(26):265602.

40. Jain TK, Foy SP, Erokwu B, Dimitrijevic S, Flask CA, Labhasetwar V. Magnetic resonance imaging of multifunctional pluronic stabilized iron-oxide nanoparticles in tumor-bearing mice. Biomaterials. 2009; 30(35):6748-6756.

41. Yallapu MM, Foy SP, Jain TK, Labhasetwar V. PEG-functionalized magnetic nanoparticles for drug delivery and magnetic resonance imaging applications. Pharm Res. 2010;27(11):2283-2295.

42. Ly JD, Grubb DR, Lawen A. The mitochondrial membrane potential (deltapsi(m)) in apoptosis; an update. Apoptosis. 2003;8(2): 115-128.

43. Manju S, Sreenivasan K. Conjugation of curcumin onto hyaluronic acid enhances its aqueous solubility and stability. $J$ Colloid Interface Sci. 2011;359(1):318-325.

44. Manju S, Sreenivasan K. Synthesis and characterization of a cytotoxic cationic polyvinylpyrrolidone-curcumin conjugate. J Pharm Sci. 2011; 100(2):504-511.

45. Marcolino VA, Zanin GM, Durrant LR, Benassi Mde T, Matioli G. Interaction of curcumin and bixin with $\beta$-cyclodextrin: complexation methods, stability, and applications in food. J Agric Food Chem. 2011; 59(7):3348-3357.

46. Hegge AB, Schuller RB, Kristensen S, Tonnesen HH. In vitro release of curcumin from vehicles containing alginate and cyclodextrin. Studies of curcumin and curcuminoides. XXXIII. Pharmazie. 2008;63(8): 585-592.

47. Shahani K, Panyam J. Highly loaded, sustained-release microparticles of curcumin for chemoprevention. J Pharm Sci. 2011;100(7): 2599-2609.

48. Utreja P, Jain S, Tiwary AK. Novel drug delivery systems for sustained and targeted delivery of anti-cancer drugs: current status and future prospects. Curr Drug Deliv. 2010;7(2):152-161.

49. Chen W, Xu N, Xu L, et al. Multifunctional magnetoplasmonic nanoparticle assemblies for cancer therapy and diagnostics (theranostics). Macromol Rapid Commun. 2010;31(2):228-236.

50. Bihari P, Vippola M, Schultes S, et al. Optimized dispersion of nanoparticles for biological in vitro and in vivo studies. Part Fibre Toxicol. 2008;5:14

51. Mahmoudi M, Laurent S, Shokrgozar MA, Hosseinkhani M. Toxicity evaluations of superparamagnetic iron oxide nanoparticles: cell "vision" versus physicochemical properties of nanoparticles. ACS Nano. 2011; 5(9):7263-7276.

52. Mahmoudi M, Simchi A, Milani AS, Stroeve P. Cell toxicity of superparamagnetic iron oxide nanoparticles. J Colloid Interface Sci. 2009;336(2):510-518 
53. Fang J, Nakamura H, Iyer AK. Tumor-targeted induction of oxystress for cancer therapy. J Drug Target. 2007;15(7-8):475-486.

54. Pelicano H, Carney D, Huang P. ROS stress in cancer cells and therapeutic implications. Drug Resist Updat. 2004;7(2):97-110.

55. Javvadi P, Segan AT, Tuttle SW, Koumenis C. The chemopreventive agent curcumin is a potent radiosensitizer of human cervical tumor cells via increased reactive oxygen species production and overactivation of the mitogen-activated protein kinase pathway. Mol Pharmacol. 2008;73(5):1491-1501.

56. Mulik RS, Monkkonen J, Juvonen RO, Mahadik KR, Paradkar AR. Transferrin mediated solid lipid nanoparticles containing curcumin: enhanced in vitro anticancer activity by induction of apoptosis. Int $J$ Pharm. 2010;398(1-2):190-203.

57. Acharya S, Sahoo SK. Sustained targeting of Bcr-Abl + leukemia cells by synergistic action of dual drug loaded nanoparticles and its implication for leukemia therapy. Biomaterials. 2011;32(24):5643-5662.

58. Misra R, Sahoo SK. Coformulation of doxorubicin and curcumin in poly(D,L-lactide-co-glycolide) nanoparticles suppresses the development of multidrug resistance in K562 cells. Mol Pharm. 2011; 8(3):852-866.
59. Raut SL, Kirthivasan B, Bommana MM, Squillante E, Sadoqi M. The formulation, characterization and in vivo evaluation of a magnetic carrier for brain delivery of NIR dye. Nanotechnology. 2010;21(39): 395102.

60. Chertok B, David AE, Yang VC. Brain tumor targeting of magnetic nanoparticles for potential drug delivery: effect of administration route and magnetic field topography. J Control Release. 2011;155(3): 393-399.

61. Child HW, Del Pino PA, De La Fuente JM, et al. Working together: the combined application of a magnetic field and penetratin for the delivery of magnetic nanoparticles to cells in 3D. ACS Nano. 2011;5(10): 7910-7919.

62. Chen YC, Chang WH, Wang SJ, Hsieh WY. Fluorescent magnetic nanoparticles with specific targeting functions for combinded targeting, optical imaging and magnetic resonance imaging. J Biomater Sci Polym Ed. 2011. [Epub ahead of print.]

63. Shan XH, Hu H, Xiong F, et al. Targeting Glut1-overexpressing MDA-MB-231 cells with 2-deoxy-d-g1ucose modified SPIOs. Eur J Radiol. 2012;81(1):95-99. 


\section{Appendix}

A

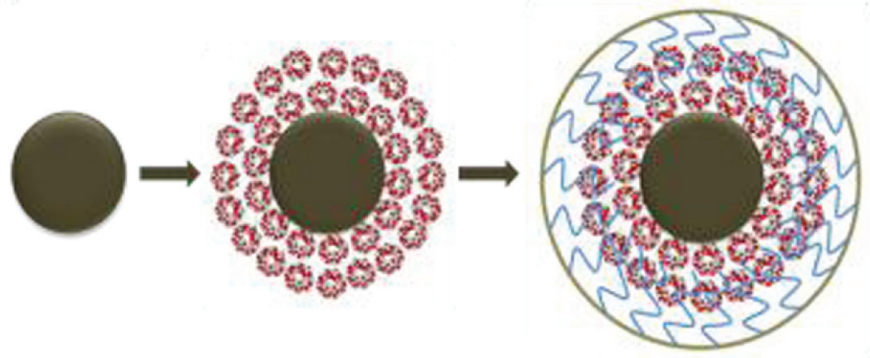

IOCNP

CD-IOCNP

F68-CD-IOCNP (MNP)

IOCNP

B

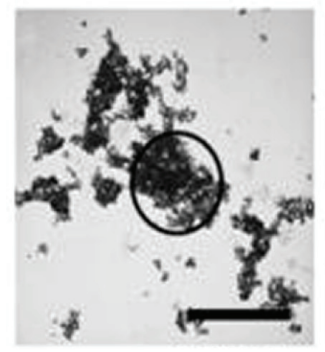

$300 \mathrm{~nm}$
CD-IOCNP

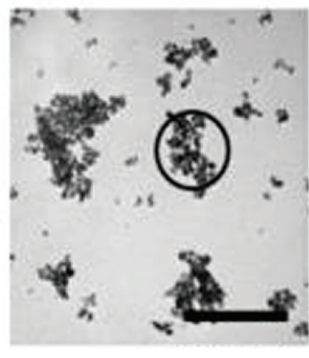

$300 \mathrm{~nm}$

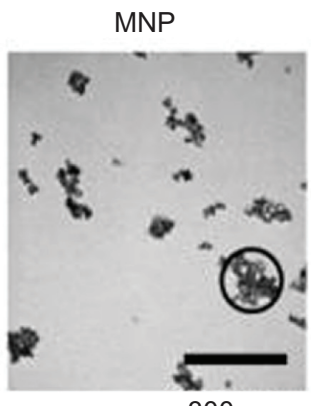

$300 \mathrm{~nm}$

C

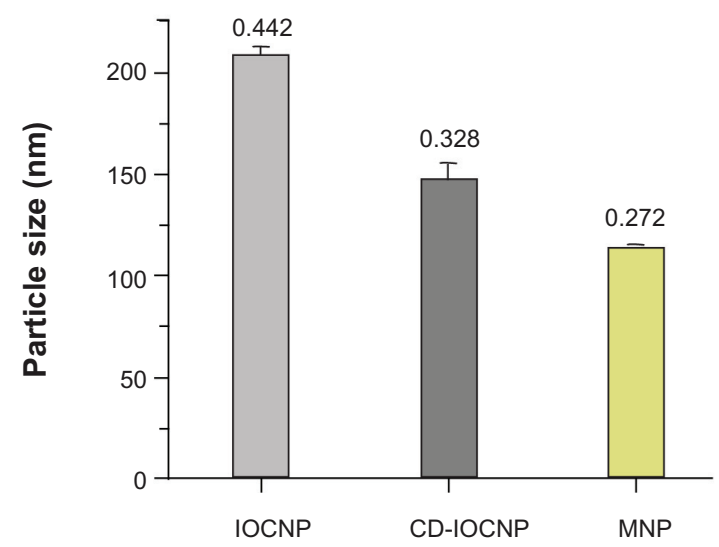

Formulations

Figure AI Composition and particle size evaluation of magnetic nanoparticles. (A) Schematic representation of the synthesis route of a multifunctional magnetic nanoparticle. Iron oxide (magnetic) nanoparticles prepared by chemical precipitation reaction in presence of $\beta$-cyclodextrin and subsequent F68 polymer coating leads to magnetic nanoparticle nanoformulation. (B) Transmission electron microscopic image of pure iron oxide core nanoparticles (no coating with $\beta$-cyclodextrin/F68 polymer), iron oxide nanoparticles coated with $100 \mathrm{mg}$ of $\beta$-cyclodextrin, and iron oxide nanoparticles coated with $100 \mathrm{mg}$ of $\beta$-cyclodextrin and $200 \mathrm{mg}$ of $\mathrm{F} 68$ polymer. Data show an individual particle grain size of $\sim 9 \mathrm{~nm}$. (C) Dynamic light scattering particle size data of (I) pure iron oxide core nanoparticles, (2) iron oxide nanoparticles coated with $\beta$-cyclodextrin, and (3) iron oxide nanoparticles coated with $\beta$-cyclodextrin and F68 polymer representing aggregative particles size in aqueous media.

Note: $\beta$-cyclodextrin and $\mathrm{F} 68$ polymer coating help to reduce particle aggregative phenomenon.

Abbreviations: CD-IOCNP, iron oxide core nanoparticles coated with CD (I00 mg); F68-CD-IOCNP/MNP, iron oxide core nanoparticles coated with CD (I00 mg) and F68 polymer (200 mg); IOCNP, iron oxide core nanoparticles. 

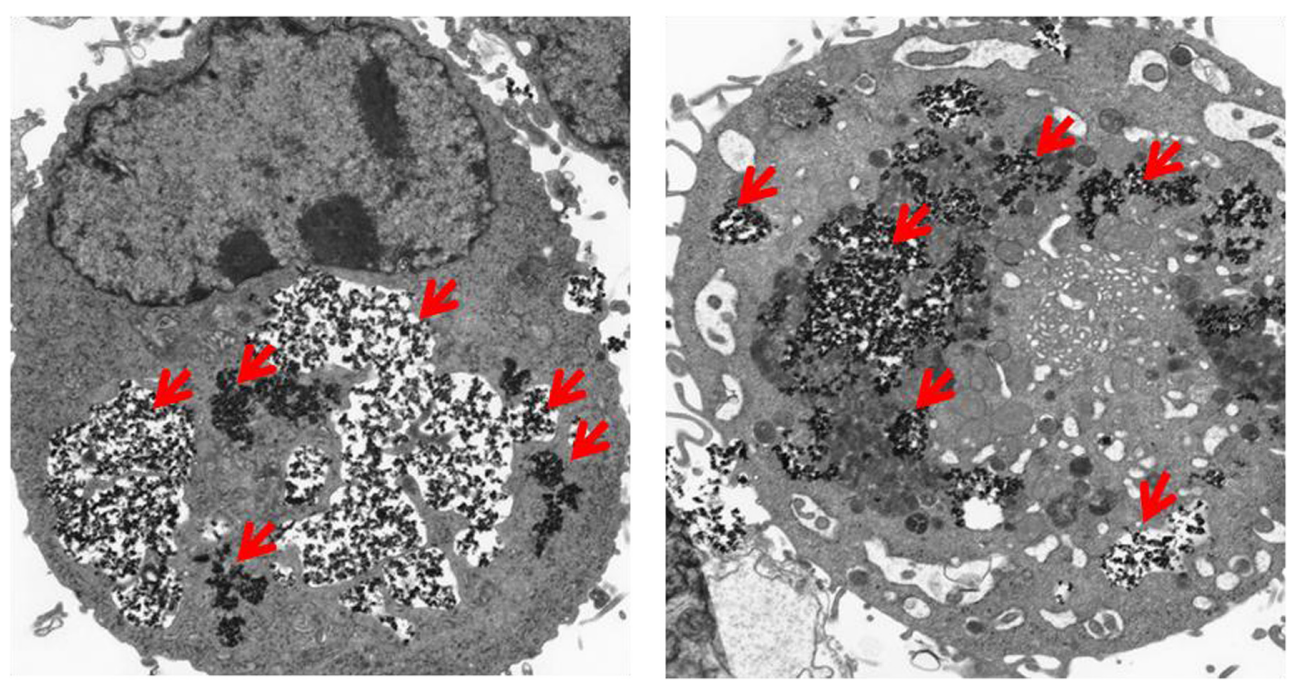

Figure A2 Magnified view of transmission electron microscopic images of MDA-MB-23I cells after treatment with curcumin-loaded magnetic nanoparticles (20 $\mu$ M) for 6 hours. Accumulation of curcumin-loaded magnetic nanoparticles occurs throughout the cytoplasm.

Note: Red arrows represent nanoparticles accumulation.

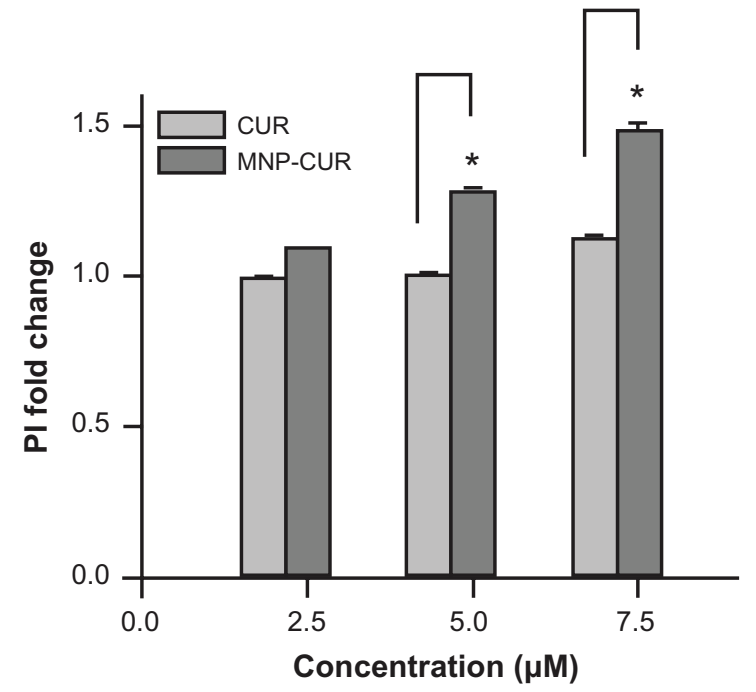

Figure A3 Curcumin-loaded magnetic nanoparticle formulation increases MDA-MB23 I cell death. Cells $\left(2 \times 10^{5}\right)$ were seeded in six-well plates and treated for 24 hours with $2.5,5$, and $7.5 \mu \mathrm{M}$ of curcumin or curcumin-loaded magnetic nanoparticles. The cells were collected both live and dead (floating) and incubated at room temperature in the dark with $5 \mu \mathrm{L}$ propidium iodide for 20 minutes in $2 \mathrm{~mL}$ medium containing hydroxyethyl piperazineethanesulfonic acid without phenol red. After completion of the incubation period, propidium iodide stain was quantified for 5000 cells using an Accuri ${ }^{\circledR}$ C6 flow cytometer (BD Accuri Cytometers, Ann Arbor, MI) in the FL2 channel. The experiment was conducted in triplicate.

Abbreviations: CUR, curcumin; MNP-CUR, curcumin-loaded nanoparticles; PI, propidium iodide.

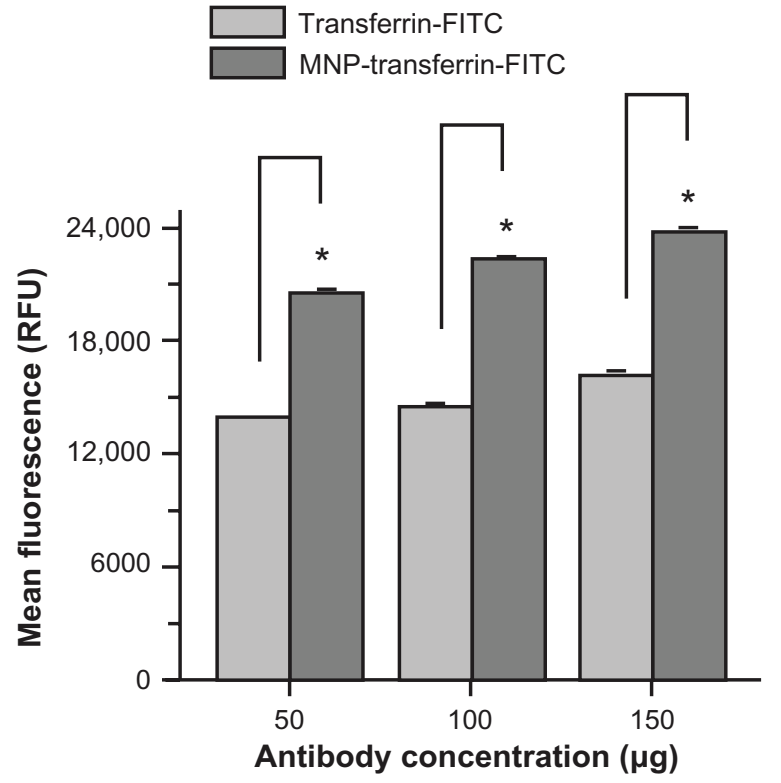

Figure A4 Antitransferrin-fluorescein isothiocyanate antibody conjugation promotes magnetic nanoparticles targeting MDA-MB-23I cancer cells. Cells $\left(2 \times 10^{5}\right)$ were seeded in six-well plates and treated for 24 hours with 50,100 , and $150 \mu \mathrm{g}$ of antitransferrinfluorescein isothiocyanate antibody or magnetic nanoparticles conjugated with antitransferrin-fluorescein isothiocyanate antibody. The cells were trypsinized and collected in I mL medium containing hydroxyethyl piperazineethanesulfonic acid without phenol red. Fluorescein isothiocyanate levels were analyzed for 5000 cells using an Accuri ${ }^{\circledast}$ C6 flow cytometer (BD Accuri Cytometers, Ann Arbor, MI) in the FLI channel. The experiment was conducted in triplicate.

Note: Antitransferrin-fluorescein isothiocyanate conjugation was performed according to previous protocol. ${ }^{19}$ In detail, $5 \mathrm{mg}$ of magnetic nanoparticles and I mg of eight-arm $\mathrm{N}$-hydroxysuccinimide-polyethylene glycol- $\mathrm{N}$-hydroxysuccinimide linker (Nanocs Inc, Boston, MA) were dispersed in I mL phosphate buffered saline. After I hour, $500 \mu \mathrm{g}$ of antitransferrin-fluorescein isothiocyanate was mixed and allowed to link onto magnetic nanoparticles overnight. To this mixture, $500 \mu \mathrm{g}$ of bovine serum albumin was added to nullify excess $\mathrm{N}$-hydroxysuccinimide reactivity. The conjugation efficiency was found to be $92 \%$.

Abbreviations: MNP-transferrin-FITC, magnetic nanoparticles conjugated with antitransferrin-fluorescein isothiocyanate antibody; RFU, relative fluorescence units; transferrin-FITC, antitransferrin-fluorescein isothiocyanate antibody. 
International Journal of Nanomedicine

Dovepress

\section{Publish your work in this journal}

The International Journal of Nanomedicine is an international, peerreviewed journal focusing on the application of nanotechnology in diagnostics, therapeutics, and drug delivery systems throughou the biomedical field. This journal is indexed on PubMed Central, MedLine, CAS, SciSearch ${ }^{\circledR}$, Current Contents ${ }^{\circledR} /$ Clinical Medicine,
Journal Citation Reports/Science Edition, EMBase, Scopus and the Elsevier Bibliographic databases. The manuscript management system is completely online and includes a very quick and fair peer-review system, which is all easy to use. Visit http://www.dovepress.com/ testimonials.php to read real quotes from published authors.

Submit your manuscript here: http://www.dovepress.com/international-journal-of-nanomedicine-journal 